\title{
Maximal Midpoint-Free Subsets of Integers
}

\author{
Roger B. Eggleton \\ School of Mathematical and Physical Sciences, University of Newcastle, Callaghan, NSW 2308, Australia
}

Correspondence should be addressed to Roger B. Eggleton; roger@ilstu.edu

Received 3 September 2014; Accepted 9 December 2014

Academic Editor: Chris A. Rodger

Copyright (C) 2015 Roger B. Eggleton. This is an open access article distributed under the Creative Commons Attribution License, which permits unrestricted use, distribution, and reproduction in any medium, provided the original work is properly cited.

A set $S \subset \mathbb{Z}$ is midpoint-free if no ordered triple $(a, b, c) \in S^{3}$ satisfies $a+c=2 b$ and $a<b<c$. Midpoint-free subsets of $\mathbb{Z}^{+}$and $\mathbb{Z}$ are studied, with emphasis on those sets characterized by restrictions on the base $m$ digits of their elements when $3 \leq m \leq 14$, and with particular attention to maximal midpoint-free subsets with $m \in\{3,4,7,9, \ldots, 13\}$.

\section{Introduction}

An ordered triple $(a, b, c)$ of integers is a midpoint triple of $\mathbb{Z}$ if $a+c=2 b$ and $a<b<c$. The midpoint of this triple is $b$, its lower endpoint is $a$ and its upper endpoint is $c$. For any subset $X \subseteq \mathbb{Z}$, let $\Lambda(X)$ denote the set of all midpoint triples $(a, b, c) \in X^{3}$. When $S \subset X \subseteq \mathbb{Z}$, let

$$
\begin{aligned}
& A(S, X):=\{a \in X \mid \exists b, c \in S:(a, b, c) \in \Lambda(X)\}, \\
& B(S, X):=\{b \in X \mid \exists a, c \in S:(a, b, c) \in \Lambda(X)\}, \\
& C(S, X):=\{c \in X \mid \exists a, b \in S:(a, b, c) \in \Lambda(X)\} .
\end{aligned}
$$

Generically, the members of $A(S, X) \cup B(S, X) \cup C(S, X)$ are the balance points for $S$ in $X$. The balance points comprise the lower endpoint set $A(S, X)$, the midpoint set $B(S, X)$, and the upper endpoint set $C(S, X)$, for $S$ in $X$. The members of $E(S, X):=X \backslash(S \cup A(S, X) \cup B(S, X) \cup C(S, X))$ are the eccentric points for $S$ in $X$. Attention to these sets appears to be a new focus, suggested by the underlying geometrical viewpoint. There is an extensive literature associated with treating $a+c=2 b$ as specifying an arithmetic progression of length 3. A compact discussion and rich bibliography are given in Guy's survey work [1, Section E10]. For an example of recent work in this field, see Dybizbański [2].

If $\Lambda(S)=\emptyset$ it will be convenient to say that $S$ is midpointfree; moreover, $S$ is a maximal midpoint-free subset of $X$ if $\Lambda(S)=\emptyset$ and $\Lambda(T) \neq \emptyset$, whenever $S \subset T \subseteq X$. Hence we have $\Lambda(S \cup\{x\}) \neq \emptyset$ for any $x \in X \backslash S$. This implies the following characterization.
Theorem 1. If $S \subset X \subseteq \mathbb{Z}$, then $S$ is a maximal midpoint-free subset of $X$ if and only if $A(S, X) \cup B(S, X) \cup C(S, X)=X \backslash S$, or equivalently, if and only if $\Lambda(S)=\emptyset$ and $E(S, X)=\emptyset$.

Note that if $X$ is infinite, any maximal midpoint-free subset $S \subset X$ must also be infinite: any pair of elements of $S$ has one midpoint and two endpoints, so precludes at most three elements of $X \backslash S$ from membership of $E(S, X)$; thus $E(S, X)$ would be infinite if $S$ were finite, but then $S \cup\{x\}$ would be midpoint-free for any $x \in E(S, X)$, contradicting maximality of $S$.

In [3] the notions of midpoint triple and maximal midpoint-free subset are studied for several "natural" subsets of the real numbers, but for simplicity in the present note we restrict $X$ to $\mathbb{Z}$ and subsets, especially $\mathbb{Z}^{+}:=\{x \in \mathbb{Z} \mid x \geq 0\}$ and $\mathbb{Z}^{-}:=\mathbb{Z} \backslash \mathbb{Z}^{+}=-\mathbb{Z}^{+} \backslash\{0\}$. Here, the main focus will be on $A(S, X), B(S, X)$, and $C(S, X)$ when $S$ is a maximal midpointfree subset of $X=\mathbb{Z}^{+}, \mathbb{Z}^{-}$or $\mathbb{Z}$.

Initially $S$ has been defined to be midpoint-free if $S^{3}$ contains no midpoint triple. There are several semantic equivalents for this condition.

Theorem 2. If $S \subset X \subseteq \mathbb{Z}$, then any one of the sets

$$
A(S, X) \cap S, \quad B(S, X) \cap S, \quad C(S, X) \cap S
$$

is empty if and only if all three are empty.

Proof. Consider the contrary. If $b \in B(S, X) \cap S$ there is a triple $(a, b, c) \in \Lambda(S)$; this triple ensures that $a \in A(S, X) \cap S$ 
and $c \in C(S, X) \cap S$. The other two cases follow in the same way.

Take $X=\mathbb{Z}$ in Theorem 2 . Then $B(S, \mathbb{Z}) \cap S=\emptyset$ recovers the "natural" terminology that $S$ is midpoint-free if and only if it does not contain the midpoint of any two of its members. Equally, $S$ is midpoint-free if and only if it is lower endpointfree, or alternatively, if and only if it is upper endpoint-free.

Corollary 3. A subset $S \subset X \subseteq \mathbb{Z}$ is midpoint-free if and only if

$$
(A(S, X) \cup B(S, X) \cup C(S, X)) \cap S=\emptyset .
$$

This yields another semantic equivalent: $S$ is midpointfree exactly when it is balance point-free.

For any $x \in X$ define the multiplicity of $x$ as a lower endpoint, midpoint, or upper endpoint for $S$, respectively, as

$$
\begin{aligned}
& \alpha(x, S):=\#\left\{(b, c) \in S^{2} \mid(x, b, c) \in \Lambda(X)\right\}, \\
& \beta(x, S):=\#\left\{(a, c) \in S^{2} \mid(a, x, c) \in \Lambda(X)\right\}, \\
& \gamma(x, S):=\#\left\{(a, b) \in S^{2} \mid(a, b, x) \in \Lambda(X)\right\} .
\end{aligned}
$$

The multiplicities for $x \in X$ in particular cases of $S \subset X \subseteq \mathbb{Z}$ will be of interest.

\section{An Explicit Example, Involving Base $m$ Digit Restrictions}

Let us begin with an explicit example to illustrate these notions and sample some of the typical features encountered.

For any integer $m \geq 3$, let $\mathbb{Z}_{m}^{+}(0,1)$ be the set of integers $x \in \mathbb{Z}^{+}$with regular base $m$ representation in which all digits are restricted to the set $\{0,1\}$. When $a, b, c$ are distinct members of $\mathbb{Z}_{m}^{+}(0,1)$, all digits of the base $m$ representation of $2 b$ lie in the set $\{0,2\}$, but base $m$ computation of $a+c$ involves no carry over, so $a+c$ contains the digit 1 in each place where the base $m$ digits of $a$ and $c$ differ. Hence $a+c=2 b$ is impossible. It follows that $\mathbb{Z}_{m}^{+}(0,1)$ is midpoint-free.

Let $(x)_{m}$ denote the regular base $m$ representation of $x$. An easy base $m$ computation shows that

$$
(112)_{m}=(111)_{m}+(1)_{m}=(101)_{m}+(11)_{m} .
$$

This corresponds to the identities

$$
m^{2}+m+2=\left(m^{2}+m+1\right)+1=\left(m^{2}+1\right)+(m+1) .
$$

Note that $m(m+1)+2$ is an even positive integer, so $z:=(1 / 2)\left(m^{2}+m+2\right)$ is a midpoint for $S:=\mathbb{Z}_{m}^{+}(0,1)$ when this set is treated as a subset of $X:=\mathbb{Z}^{+}$. Digit considerations make it clear that $2 z=(112)_{m}=(a)_{m}+(c)_{m}$ has no other solutions with $a, c \in \mathbb{Z}_{m}^{+}(0,1)$, so the midpoint multiplicity of $z$ is 2 . In summary,

$$
\begin{gathered}
z:=\frac{1}{2}\left(m^{2}+m+2\right) \in B\left(\mathbb{Z}_{m}^{+}(0,1), \mathbb{Z}^{+}\right), \\
\beta\left(z, \mathbb{Z}_{m}^{+}(0,1)\right)=2 .
\end{gathered}
$$

Again, the general base $m$ computation

$$
(112)_{m}+(110)_{m}=(222)_{m}=2 \cdot(111)_{m}
$$

corresponds to the identity

$$
\left(m^{2}+m+2\right)+\left(m^{2}+m\right)=2\left(m^{2}+m+1\right),
$$

which implies

$$
\begin{gathered}
2 z=m^{2}+m+2 \in C\left(\mathbb{Z}_{m}^{+}(0,1), \mathbb{Z}^{+}\right), \\
\gamma\left(2 z, \mathbb{Z}_{m}^{+}(0,1)\right)=1,
\end{gathered}
$$

when $m \geq 4$. Additionally, in the special case $m=3$ we also have

$$
\begin{gathered}
(112)_{3}+(11)_{3}=2 \cdot(100)_{3} ; \\
(112)_{3}+(101)_{3}=2 \cdot(110)_{3} ; \\
(112)_{3}+(1111)_{3}=2 \cdot(1000)_{3} .
\end{gathered}
$$

Hence $(112)_{3}=14 \in A\left(\mathbb{Z}_{3}^{+}(0,1), \mathbb{Z}^{+}\right) \cap C\left(\mathbb{Z}_{3}^{+}(0,1), \mathbb{Z}^{+}\right)$. There are no other solutions to $(112)_{3}+(c)_{3}=2 \cdot(b)_{3}$ with $b, c \in \mathbb{Z}_{3}^{+}(0,1)$, so the multiplicities of 14 as a lower and upper endpoint are $\alpha\left(14, \mathbb{Z}_{3}^{+}(0,1)\right)=1, \gamma\left(14, \mathbb{Z}_{3}^{+}(0,1)\right)=3$.

When $m \geq 5$, it can be seen that $(24)_{m}+(c)_{m}=2 \cdot(b)_{m}$ has no solutions with $b, c \in \mathbb{Z}_{m}^{+}(0,1)$, so $2 m+4$ is not an endpoint for $\mathbb{Z}_{m}^{+}(0,1)$, and

$$
\alpha\left(2 m+4, \mathbb{Z}_{m}^{+}(0,1)\right)=\gamma\left(2 m+4, \mathbb{Z}_{m}^{+}(0,1)\right)=0 .
$$

Now seek $a, c \in \mathbb{Z}_{m}^{+}(0,1)=\left\{0,1, m, m+1, m^{2}, m^{2}+1\right.$, $\left.m^{2}+m, m^{2}+m+1, m^{3}, \ldots\right\}$ such that $a+c=2(2 m+4)$ and $a<2 m+4<c$. As $m^{2}>2 m+4$, it follows that $a \in\{0,1, m, m+1\}$. Then it is routine to verify that

$$
c \in\{3 m+7,3 m+8,4 m+7,4 m+8\} \cap \mathbb{Z}_{m}^{+}(0,1)=\emptyset
$$

when $m \geq 5$, but note that digit arguments must be sensitive to the magnitude of $m$; for instance, $4 m+7=(47)_{m}$ when $m \geq 8$, while for smaller $m$ we have

$$
\begin{gathered}
4 m+7=m^{2}+m+3=(113)_{m} \quad \text { when } m=4, \\
4 m+7=m^{2}+2=(102)_{m} \quad \text { when } m=5, \\
4 m+7=5 m+1=(51)_{m} \quad \text { when } m=6, \\
4 m+7=5 m=(50)_{m} \quad \text { when } m=7 .
\end{gathered}
$$

It follows that

$$
\beta\left(2 m+4, \mathbb{Z}_{m}^{+}(0,1)\right)=0,
$$

and $2 m+4$ is not a midpoint for $\mathbb{Z}_{m}^{+}(0,1)$ when $m \geq 5$. This completes the demonstration that $2 m+4$ is an eccentric point, so the midpoint-free subset $\mathbb{Z}_{m}^{+}(0,1) \subset \mathbb{Z}^{+}$is not maximal if $m \geq 5$.

We will later return to a more systematic study of $\mathbb{Z}_{3}^{+}(0,1)$ and $\mathbb{Z}_{4}^{+}(0,1)$. 


\section{New Maximal Midpoint-Free Sets from Old}

If $S \subset \mathbb{Z}$ and $c, d \in \mathbb{Z}$ with $c \neq 0$, then $c S+d:=\{c s+d \mid s \in S\}$ is an affine transform of $S$. Clearly $(x, y, z) \in \Lambda(\mathbb{Z})$ if and only if $c(x, y, z)+d \in \Lambda(\mathbb{Z})$ when $c>0$ or $c(z, y, x)+d \in \Lambda(\mathbb{Z})$ when $c<0$, so any affine transform $c S+d$ is midpoint-free if and only if $S$ is midpoint-free.

For example, let $\mathbb{Z}_{m}^{+}(0,2)$ be the set of all integers $x \in \mathbb{Z}^{+}$ with regular base $m \geq 3$ representation in which all digits are restricted to the set $\{0,2\}$. Then $\mathbb{Z}_{m}^{+}(0,2)$ is midpointfree because $\mathbb{Z}_{m}^{+}(0,1)$ is midpoint-free when $m \geq 3$, and $\mathbb{Z}_{m}^{+}(0,2)=2 \mathbb{Z}_{m}^{+}(0,1)$ is an affine transform of $\mathbb{Z}_{m}^{+}(0,1)$. The last identity shows that $\mathbb{Z}_{m}^{+}(0,2) \subset 2 \mathbb{Z}^{+}$. Similarly, $-\left(\mathbb{Z}_{m}^{+}(0,2)+1\right)=-2 \mathbb{Z}_{m}^{+}(0,1)-1$ is an affine transform of $\mathbb{Z}_{m}^{+}(0,1)$ so is a midpoint-free subset of $2 \mathbb{Z}^{-}+1$ when $m \geq 3$. Then

$$
\mathbb{Z}_{m}^{+}(0,2) \cup-\left(\mathbb{Z}_{m}^{+}(0,2)+1\right)
$$

is a midpoint-free subset of $\mathbb{Z}$, since the positive and negative components of this set are midpoint-free, and $a+c=2 b$ has no solution with $a \in 2 \mathbb{Z}^{-}+1, c \in 2 \mathbb{Z}^{+}$, and $b \in \mathbb{Z}$, because these conditions require $a+c$ to be an odd integer and $2 b$ to be an even integer.

Suppose $S \subset \mathbb{Z}^{+}$is known to be a maximal midpoint-free subset of $\mathbb{Z}$. It turns out that the principle illustrated by the example in the previous paragraph holds strongly for $S$.

Theorem 4. If $\subset \subset \mathbb{Z}^{+}$is a maximal midpoint-free subset of $\mathbb{Z}$, then the set $S^{(2)}:=2 S \cup-(2 S+1)$ is a maximal midpoint-free subset of $\mathbb{Z}$.

Proof. As $S$ is a maximal midpoint-free subset of $\mathbb{Z}$, Theorem 1 implies

$$
A(S, \mathbb{Z}) \cup B(S, \mathbb{Z}) \cup C(S, \mathbb{Z})=\mathbb{Z} \backslash S .
$$

But $S \subset \mathbb{Z}^{+}$, so $B(S, \mathbb{Z}) \subset \mathbb{Z}^{+}$and $C(S, \mathbb{Z}) \subset \mathbb{Z}^{+}$; hence $B(S, \mathbb{Z})=B\left(S, \mathbb{Z}^{+}\right)$and $C(S, \mathbb{Z})=C\left(S, \mathbb{Z}^{+}\right)$. Therefore, $A(S, \mathbb{Z})=A\left(S, \mathbb{Z}^{+}\right) \cup \mathbb{Z}^{-}$.

Given $x \in A(S, \mathbb{Z}) \cup C(S, \mathbb{Z})$, there exist $b, y \in S$ such that $x+y=2 b$ and $b \neq y$. Let $\delta \in\{0,1\}$. Then $(2 x+\delta)+(2 y+\delta)=$ $2(2 b+\delta)$, so

$$
\begin{aligned}
& x<y \Longrightarrow 2 x+\delta \in A(2 S+\delta, \mathbb{Z}) ; \\
& x>y \Longrightarrow 2 x+\delta \in C(2 S+\delta, \mathbb{Z}) .
\end{aligned}
$$

Therefore,

$$
\begin{aligned}
& 2 A(S, \mathbb{Z})+\delta \subseteq A(2 S+\delta, \mathbb{Z}), \\
& 2 C(S, \mathbb{Z})+\delta \subseteq C(2 S+\delta, \mathbb{Z}) .
\end{aligned}
$$

Conversely, suppose $u \in A(2 S+\delta, \mathbb{Z}) \cup C(2 S+\delta, \mathbb{Z})$, so there exist $d, v \in 2 S+\delta$ such that $u+v=2 d$ and $d \neq v$. But $d=2 d^{\prime}+\delta, v=2 v^{\prime}+\delta$ where $d^{\prime}, v^{\prime} \in S$, so $u+v \equiv 0 \bmod 2$ implies $u \equiv v \equiv \delta \bmod 2$, whence $u=2 u^{\prime}+\delta$ with $u^{\prime} \in \mathbb{Z}$. But $S$ is midpoint-free, so $2 S+\delta$ is midpoint-free, and therefore, $u \in \mathbb{Z} \backslash(2 S+\delta)$. Thus $u^{\prime} \in \mathbb{Z} \backslash S$. Now $u+v=2 d$ and $d \neq v$ imply that $u^{\prime}+v^{\prime}=2 d^{\prime}$ and $d^{\prime} \neq v^{\prime}$, so $u^{\prime} \in A(S, \mathbb{Z}) \cup C(S, \mathbb{Z})$. Therefore, the reverse containments also hold:

$$
\begin{aligned}
& u<v \Longrightarrow u \in 2 A(S, \mathbb{Z})+\delta ; \\
& u>v \Longrightarrow u \in 2 C(S, \mathbb{Z})+\delta .
\end{aligned}
$$

Hence

$$
\begin{aligned}
& A(2 S+\delta, \mathbb{Z})=2 A(S, \mathbb{Z})+\delta, \\
& C(2 S+\delta, \mathbb{Z})=2 C(S, \mathbb{Z})+\delta .
\end{aligned}
$$

For $x \in B(S, \mathbb{Z})$, similar reasoning shows that $2 x+\delta \in$ $B\left(2 S+\delta, \mathbb{Z}^{+}\right)$, so

$$
2 B(S, \mathbb{Z})+\delta \subseteq B(2 S+\delta, \mathbb{Z}) .
$$

However, this containment can in fact be proper. For instance, if $S=\left\{2^{n} \mid n \in \mathbb{Z}^{+}\right\}$, then $2 B(S, \mathbb{Z})$ only contains even integers, whereas $\left\{2^{n+1}+1 \mid n \in \mathbb{Z}^{+}\right\} \subset B(2 S, \mathbb{Z})$.

Since $S$ is a maximal midpoint-free subset of $\mathbb{Z}$, no integers are eccentric for $S$, so no members of $2 \mathbb{Z}^{+}+\delta$ are eccentric for $2 S+\delta$. Then the positive integers eccentric for $2 S+\delta$ satisfy

$$
E\left(2 S+\delta, \mathbb{Z}^{+}\right)=\left(2 \mathbb{Z}^{+}+\varepsilon\right) \backslash B(2 S+\delta, \mathbb{Z}) \subseteq 2 \mathbb{Z}^{+}+\varepsilon,
$$

where $\varepsilon:=1-\delta$. Since $A(S, \mathbb{Z})=A\left(S, \mathbb{Z}^{+}\right) \cup \mathbb{Z}^{-}$it follows that

$$
E(2 S+\delta, \mathbb{Z}) \subseteq 2 \mathbb{Z}+\varepsilon .
$$

Specifically, all integers eccentric for $2 S$ are odd, and all integers eccentric for $2 S+1$ are even. Therefore, no integer is eccentric for both $2 S$ and $-(2 S+1)$, so $E\left(S^{(2)}, \mathbb{Z}\right)=\emptyset$. Thus $S^{(2)}$ is a maximal midpoint-free subset of $\mathbb{Z}$.

Corollary 5. If $S \subset \mathbb{Z}^{+}$is a maximal midpoint-free subset of $\mathbb{Z}$, the balance point sets for $S$ and $2 S+\delta$ with $\delta \in\{0,1\}$ satisfy

$$
\begin{aligned}
& A(S, \mathbb{Z})= A\left(S, \mathbb{Z}^{+}\right) \cup \mathbb{Z}^{-}, \quad B(S, \mathbb{Z})=B\left(S, \mathbb{Z}^{+}\right), \\
& C(S, \mathbb{Z})=C\left(S, \mathbb{Z}^{+}\right), \quad A(2 S+\delta, \mathbb{Z})=2 A(S, \mathbb{Z})+\delta, \\
& C(2 S+\delta, \mathbb{Z})=2 C(S, \mathbb{Z})+\delta, \\
& B(2 S+\delta, \mathbb{Z}) \supseteq 2 B(S, \mathbb{Z})+\delta .
\end{aligned}
$$

It is convenient to refer to the construction in Theorem 4 as "doubling" the given set $S$. Other constructions involving affine transforms of a set are also of interest. For example, since $\mathbb{Z}_{m}^{+}(0,1)$ is midpoint-free when $m \geq 3$, it follows that the disjoint sets $m \mathbb{Z}_{m}^{+}(0,1)$ and $m \mathbb{Z}_{m}^{+}(0,1)+1$ are midpoint-free when $m \geq 3$. In fact, their union is midpointfree. This turns out to be "trivial." Multiplying a member of $\mathbb{Z}_{m}^{+}(0,1)$ by $m$ simply shifts its base $m$ digits one place, and a terminal 0 emerges to occupy the zeroth place; then adding 1 replaces the terminal 0 by 1 . Hence $m \mathbb{Z}_{m}^{+}(0,1) \cup$ $\left(m \mathbb{Z}_{m}^{+}(0,1)+1\right)=\mathbb{Z}_{m}^{+}(0,1)$.

When $m \geq 4$, the disjoint midpoint-free sets $3 \mathbb{Z}_{m}^{+}(0,1)$ and $3 \mathbb{Z}_{m}^{+}(0,1)+1$ are more interesting. Let us verify that

$$
S:=3 \mathbb{Z}_{m}^{+}(0,1) \cup\left(3 \mathbb{Z}_{m}^{+}(0,1)+1\right)
$$


is also midpoint-free. The two component sets are midpointfree, so any midpoint triple $(a, b, c) \in \Lambda(S)$ must have at least one member in each set. Thus $\{a, b, c\} \cap\left(3 \mathbb{Z}^{+}+\delta\right) \neq \emptyset$ in each case with $\delta \in\{0,1\}$. If $\{a, c\} \subset 3 \mathbb{Z}_{m}^{+}(0,1)+\delta$, then $b \in 3 \mathbb{Z}_{m}^{+}(0,1)+\varepsilon$ for $\varepsilon:=1-\delta \in\{0,1\}$. Then $a+c \in 3 \mathbb{Z}^{+}+2 \delta$ and $2 b \in 3 \mathbb{Z}^{+}+2 \varepsilon$. But $\{2 \delta, 2 \varepsilon\}=\{0,2\}$, so $a+c \neq 2 b$. If $\{a, b\} \subset 3 \mathbb{Z}_{m}^{+}(0,1)+\delta$ then $c \in 3 \mathbb{Z}_{m}^{+}(0,1)+\varepsilon$, while if $\{b, c\} \subset 3 \mathbb{Z}_{m}^{+}(0,1)+\delta$ then $a \in 3 \mathbb{Z}_{m}^{+}(0,1)+\varepsilon$. In each case $a+c \in 3 \mathbb{Z}^{+}+1$ and $2 b \in 3 \mathbb{Z}^{+}+2 \delta$, so $a+c \neq 2 b$ because $2 \delta \in\{0,2\}$. Thus $\Lambda(S)=\emptyset$, as claimed.

Generalising the latter example, a "trebling" construction which produces new maximal midpoint-free subsets of $\mathbb{Z}$ will now be studied.

Theorem 6. If $S \subset \mathbb{Z}^{+}$is a maximal midpoint-free subset of $\mathbb{Z}$, and all members of $\mathbb{Z}^{+} \backslash S$ are endpoints for $S$, then $S^{(3)}:=3 S \cup(3 S+1)$ is a maximal midpoint-free subset of $\mathbb{Z}$, and all members of $\mathbb{Z} \backslash S^{(3)}$ are endpoints of $S^{(3)}$.

Proof. Because $S$ is midpoint-free, each of the affine transforms $3 S$ and $3 S+1$ is midpoint-free. Assume $(a, b, c) \in$ $\Lambda\left(S^{(3)}\right)$. Since $S^{(3)} \subset 3 \mathbb{Z}^{+} \cup\left(3 \mathbb{Z}^{+}+1\right)$, there is a $\delta \in\{0,1\}$ such that $b \in 3 \mathbb{Z}^{+}+\delta$. Then $a+c=2 b \in 3 \mathbb{Z}^{+}+2 \delta$, so $a, b, c \in 3 \mathbb{Z}^{+}+\delta$. Thus $(a, b, c) \in \Lambda(3 S+\delta)=\emptyset$, so no such triple exists. Hence $S^{(3)}:=3 S \cup(3 S+1)$ is midpoint-free.

By hypothesis, $A\left(S, \mathbb{Z}^{+}\right) \cup C\left(S, \mathbb{Z}^{+}\right)=\mathbb{Z}^{+} \backslash S$. Also $A(S, \mathbb{Z})=A\left(S, \mathbb{Z}^{+}\right) \cup \mathbb{Z}^{-}$by Corollary 5 , so every $x \in \mathbb{Z} \backslash S$ is an endpoint for $S$. Suppose $x+y=2 b$ and $b, y \in S$. Two integers from complementary sets cannot be equal, so $x, y, b$ must be different. Also

$$
(3 x+r)+(3 y+t)=2(3 b+s)
$$

holds when $(r, s, t) \in\{(0,0,0),(1,1,1),(2,1,0),(-1,0,1)\}$. It follows that

$$
\begin{gathered}
x<y \Longrightarrow x \in A(S, \mathbb{Z}), \\
3 x+\{-1,0,1,2\} \subset A\left(S^{(3)}, \mathbb{Z}\right), \\
x>y \Longrightarrow x \in C(S, \mathbb{Z}), \\
3 x+\{-1,0,1,2\} \subset C\left(S^{(3)}, \mathbb{Z}\right) .
\end{gathered}
$$

If $s<s^{\prime}$ are consecutive members of $S$, all members of the interval

$$
\left[s+1, s^{\prime}-1\right]:=\left\{z \in \mathbb{Z} \mid s+1 \leq z \leq s^{\prime}-1\right\}
$$

are endpoints for $S$. Then $3 s+1<3 s^{\prime}$ are consecutive members of $S^{(3)}$ and all members of $\left[3(s+1)-1,3\left(s^{\prime}-1\right)+2\right]=$ $\left[3 s+2,3 s^{\prime}-1\right]$ are endpoints for $S^{(3)}$. Also each $x \in \mathbb{Z}^{-}$is an endpoint for $S$, so all members of $[3 x-1,3 x+2]$ are endpoints for $S^{(3)}$. Thus $A\left(S^{(3)}, \mathbb{Z}\right) \cup C\left(S^{(3)}, \mathbb{Z}\right)=\mathbb{Z} \backslash S^{(3)}$, and $E\left(S^{(3)}, \mathbb{Z}\right)=\emptyset$.

Let $U_{n}:=\left\{x \in \mathbb{Z}_{3}^{+}(0,1) \mid 0 \leq x<3^{n}\right\}$ for each $n \in \mathbb{Z}^{+}$, and let $S^{(3,0)}:=S$. Iterating the construction in Theorem 6 and combining with Theorem 4 yields the following result.
Corollary 7. If $S \subset \mathbb{Z}^{+}$is a maximal midpoint-free subset of $\mathbb{Z}$, and all members of $\mathbb{Z}^{+} \backslash S$ are endpoints for $S$, then the set

$$
S^{(3, n)}:=\bigcup_{x \in U_{n}}\left(3^{n} S+x\right)
$$

is a maximal midpoint-free subset of $\mathbb{Z}$, for any integer $n \in \mathbb{Z}^{+}$, and all members of $\mathbb{Z} \backslash S^{(3, n)}$ are endpoints of $S^{(3, n)}$. Moreover, the set

$$
S^{(2,3, n)}:=2 S^{(3, n)} \cup-\left(2 S^{(3, n)}+1\right)
$$

is a maximal midpoint-free subset of $\mathbb{Z}$.

\section{Subsets of $\mathbb{Z}^{+}$with Base $m$ Digit Restrictions}

Fix an integer $m \geq 3$. Let $\{0\} \subseteq D \subset\left\{x \in \mathbb{Z}^{+} \mid 0 \leq x<m\right\}:=$ $[0, m)$. Then $D$ is a digit subset for base $m$ representations of the integers or, briefly, a base $m$ digit subset. Let $\mathbb{Z}_{m}^{+}(D)$ be the set of nonnegative integers with base $m$ representation using only digits in $D$, and let $\llbracket x \rrbracket_{m, i}$ denote the digit in position $i \geq 0$ of the regular base $m$ representation of $x \in \mathbb{Z}^{+}$, so

$$
\mathbb{Z}_{m}^{+}(D):=\left\{x \in \mathbb{Z}^{+} \mid \forall i \in \mathbb{Z}^{+}: \llbracket x \rrbracket_{m, i} \in D\right\}
$$

Let us say that $D$ is midpoint-free as a base $m$ digit subset if $2 \cdot \max (D)<m$ and there is no ordered triple $(d, e, f) \in D^{3}$ such that $d \neq f$ and $d+f=2 e$.

Theorem 8. If $D$ is a midpoint-free base $m$ digit subset with $g:=\max D \geq 1$ and $m \geq 2 g+1$, then the set $\mathbb{Z}_{m}^{+}(D)$ is midpoint-free.

Proof. Suppose $(a, b, c) \in \Lambda\left(\mathbb{Z}_{m}^{+}(D)\right)$. Then $a+c=2 b$. There is no carry-over in computing this sum in base $m$ arithmetic since all its digits are less than $m / 2$, so

$$
\llbracket a \rrbracket_{m, i}+\llbracket c \rrbracket_{m, i}=\llbracket 2 b \rrbracket_{m, i}=2 \llbracket b \rrbracket_{m, i}
$$

for every $i \geq 0$. Since $D$ is midpoint-free, it follows that

$$
\llbracket a \rrbracket_{m, i}=\llbracket b \rrbracket_{m, i}=\llbracket c \rrbracket_{m, i}
$$

for every $i \geq 0$, so $a=b=c$, contradicting the initial choice of $(a, b, c)$. Thus $\Lambda\left(\mathbb{Z}_{m}^{+}(D)\right)=\emptyset$, so $\mathbb{Z}_{m}^{+}(D)$ is midpoint-free.

Three early instances of Theorem 8, the first of which was independently demonstrated earlier, are of considerable interest.

Corollary 9. Each $\mathbb{Z}_{m}^{+}(0,1)$ is midpoint-free when $m \geq 3$.

Corollary 10. Each $\mathbb{Z}_{m}^{+}(0,1,3)$ is midpoint-free when $m \geq 7$.

Corollary 11. Each $\mathbb{Z}_{m}^{+}(0,1,3,4)$ is midpoint-free when $m \geq 9$.

If $D$ is a midpoint-free base $m \geq 3$ digit subset, it is of interest to decide whether $\mathbb{Z}^{+}$has any members that are eccentric for $\mathbb{Z}_{m}^{+}(D)$, since this is equivalent to deciding whether $\mathbb{Z}_{m}^{+}(D)$ is a maximal midpoint-free subset of $\mathbb{Z}^{+}$. 
For $x \in \mathbb{Z}^{+}$, let the support for $x$, as a lower endpoint, midpoint, or upper endpoint for $S$, be defined by

$$
\begin{aligned}
& \operatorname{supp}_{A}\left(x, \mathbb{Z}_{m}^{+}(D)\right) \\
&:=\bigcup\left\{b, c \in \mathbb{Z}_{m}^{+}(D) \mid(x, b, c) \in \Lambda\left(\mathbb{Z}^{+}\right)\right\}, \\
& \operatorname{supp}_{B}\left(x, \mathbb{Z}_{m}^{+}(D)\right) \\
&:=\bigcup\left\{a, c \in \mathbb{Z}_{m}^{+}(D) \mid(a, x, c) \in \Lambda\left(\mathbb{Z}^{+}\right)\right\}, \\
& \operatorname{supp}_{C}\left(x, \mathbb{Z}_{m}^{+}(D)\right) \\
&:=\bigcup\left\{a, b \in \mathbb{Z}_{m}^{+}(D) \mid(a, b, x) \in \Lambda\left(\mathbb{Z}^{+}\right)\right\} .
\end{aligned}
$$

The following result is useful for settling whether the eccentric set $E(S, X)$ is empty in specific cases.

Theorem 12. Suppose $m \geq 3$ and $D$ is a midpoint-free base $m$ digit subset. If $x \in\left[0, m^{k}\right)$ with $k \geq 1$, then

$$
\begin{aligned}
& \operatorname{supp}_{A}\left(x, \mathbb{Z}_{m}^{+}(D)\right) \subset\left[0, m^{k+1}\right) \cap \mathbb{Z}_{m}^{+}(D), \\
& \operatorname{supp}_{B}\left(x, \mathbb{Z}_{m}^{+}(D)\right) \subset\left[0,2 m^{k}\right) \cap \mathbb{Z}_{m}^{+}(D), \\
& \operatorname{supp}_{C}\left(x, \mathbb{Z}_{m}^{+}(D)\right) \subset\left[0, m^{k}\right) \cap \mathbb{Z}_{m}^{+}(D) .
\end{aligned}
$$

Proof. Fix the nonnegative integer $x \in\left[0, m^{k}\right)$ with $k \geq 1$. (For simplicity we do not explicitly require $x \notin \mathbb{Z}_{m}^{+}(D)$, although Theorem 8 does imply that members of $\mathbb{Z}_{m}^{+}(D)$ have empty support sets.)

Suppose there exist $b, c \in \mathbb{Z}_{m}^{+}(D)$ such that $(x, b, c) \in$ $\Lambda\left(\mathbb{Z}^{+}\right)$, so $x+c=2 b$. Base $m$ arithmetic for this sum takes the form

$$
\llbracket x \rrbracket_{m, i}+\llbracket c \rrbracket_{m, i}+\delta_{i}=\llbracket 2 b \rrbracket_{m, i}+m \delta_{i+1}
$$

for every $i \geq 0$, with carry-overs $\delta_{i} \in\{0,1\}$ satisfying $\delta_{0}=0$ and

$$
\begin{gathered}
\llbracket x \rrbracket_{m, i}+\llbracket c \rrbracket_{m, i}+\delta_{i}<m \Longrightarrow \delta_{i+1}=0 \\
\llbracket x \rrbracket_{m, i}+\llbracket c \rrbracket_{m, i}+\delta_{i} \geq m \Longrightarrow \delta_{i+1}=1
\end{gathered}
$$

for $i>0$. But $x<m^{k}$ so $\llbracket x \rrbracket_{m, i}=0$ for $i \geq k$. In particular,

$$
\llbracket x \rrbracket_{m, k}+\llbracket c \rrbracket_{m, k}+\delta_{k}<\frac{m}{2}+1<m
$$

so $\delta_{k+1}=0$. For all $i \geq k+1$, it follows that $\delta_{i}=0$ and

$$
\llbracket x \rrbracket_{m, i}+\llbracket c \rrbracket_{m, i}+\delta_{i}=\llbracket c \rrbracket_{m, i}=\llbracket 2 b \rrbracket_{m, i}=2 \llbracket b \rrbracket_{m, i} .
$$

But $D$ is midpoint-free, so $\llbracket b \rrbracket_{m, i}=\llbracket c \rrbracket_{m, i}=0$ for all $i \geq$ $k+1$, since otherwise $\left(0, \llbracket b \rrbracket_{m, i}, \llbracket c \rrbracket_{m, i}\right) \in \Lambda(D)$ yields the contradiction $\Lambda(D) \neq \emptyset$. Hence $b<c<m^{k+1}$.

The other two cases follow simply by noting that if $(a, x, c) \in \Lambda\left(\mathbb{Z}^{+}\right)$then $a<c \leq a+c=2 x<2 m^{k}$, and if $(a, b, x) \in \Lambda\left(\mathbb{Z}^{+}\right)$then $a<b<x<m^{k}$.
Corollary 13. If $D$ is a midpoint-free base $m \geq 3$ digit subset, then relative to $\mathbb{Z}_{m}^{+}(D)$ the three multiplicities of any $x \in \mathbb{Z}^{+}$ are finite.

Corollary 14. If $D$ is a midpoint-free base $m \geq 3$ digit subset and $x \in\left[0, m^{k}\right)$ with $k \geq 1$, then $M_{k}:=\left[0, m^{k+1}\right) \cap$ $\mathbb{Z}_{m}^{+}(D)$ contains all three support sets for $x$ relative to $\mathbb{Z}_{m}^{+}(D)$. Moreover $\# M_{k}=d^{k+1}$, where $d:=\# D$.

Corollary 15. If $m \geq 5$ then $m+3$ is eccentric for $\mathbb{Z}_{m}^{+}(0,1)$.

Corollary 16. If $m \geq 8$ then $2 m+4$ is eccentric for $\mathbb{Z}_{m}^{+}(0,1,3)$.

Corollary 17. If $m \geq 14$ then $4 m+9$ is eccentric for $\mathbb{Z}_{m}^{+}(0,1,3,4)$.

The last three corollaries leave open the possibility that their subject sets $\mathbb{Z}_{m}^{+}(D)$ might be maximal midpoint-free subsets of $\mathbb{Z}^{+}$when $m$ is small enough. In the next section we shall consider $\mathbb{Z}_{3}^{+}(0,1)$ and pursue other cases later.

\section{Greedy Midpoint-Free Subset of $\mathbb{Z}^{+}$}

The greedy midpoint-free subset of $\mathbb{Z}^{+}$is the set

$$
S_{0}:=\left\{s_{i} \in \mathbb{Z}^{+} \mid i \in \mathbb{Z}^{+}\right\}
$$

in which $s_{0}=0$ and each $s_{i}$ with $i>0$ is the smallest integer satisfying $s_{i}>s_{i-1}$ such that $\left\{s_{0}, s_{1}, \ldots, s_{i}\right\}$ is midpoint-free. It has long been known [4] that

$$
S_{0}=\mathbb{Z}_{3}^{+}(0,1)=\{0,1,3,4,9,10,12,13,27, \ldots\},
$$

corresponding to sequence A00536 of OEIS [5]. For brevity, let

$$
\begin{gathered}
A_{0}^{+}:=A\left(S_{0}, \mathbb{Z}^{+}\right), \quad B_{0}^{+}:=B\left(S_{0}, \mathbb{Z}^{+}\right), \\
C_{0}^{+}:=C\left(S_{0}, \mathbb{Z}^{+}\right) .
\end{gathered}
$$

We shall attach the adjective greedy when referring to these sets. Before we prove that each of these greedy balance point sets contains all positive integers not in $\mathbb{Z}_{3}^{+}(0,1)$, let us check the example $32=(1012)_{3} \in \mathbb{Z}^{+} \backslash \mathbb{Z}_{3}^{+}(0,1)$. The following base 3 computations are transparent:

$$
\begin{aligned}
(1012)_{3}+(1010)_{3} & =2 \cdot(1011)_{3}, \\
(1012)_{3}+(1111)_{3} & =2 \cdot(1100)_{3}, \\
2 \cdot(1012)_{3}=(2101)_{3} & =(1000)_{3}+(1101)_{3} .
\end{aligned}
$$

Hence we have the midpoint triples $(30,31,32),(32,36,40)$, $(27,32,37) \in \Lambda\left(\mathbb{Z}^{+}\right)$, showing that $32 \in A_{0}^{+} \cap B_{0}^{+} \cap C_{0}^{+}$. Now consider the general case.

Theorem 18. The greedy midpoint-free subset $\mathbb{Z}_{3}^{+}(0,1) \subset \mathbb{Z}^{+}$ has greedy balance point sets satisfying

$$
A_{0}^{+}=B_{0}^{+}=C_{0}^{+}=\mathbb{Z}^{+} \backslash \mathbb{Z}_{3}^{+}(0,1) .
$$


Proof. Let $x \in \mathbb{Z}^{+} \backslash \mathbb{Z}_{3}^{+}(0,1)$. First we show that $x$ is a midpoint for $\mathbb{Z}_{3}^{+}(0,1)$. Note that there is at least one $j \geq 0$ such that $\llbracket 2 x \rrbracket_{3, j}=1$. Specify $a, c \in \mathbb{Z}_{3}^{+}(0,1)$ by their base 3 digits; thus

$$
\begin{aligned}
& \llbracket 2 x \rrbracket_{3, i}=1 \Longrightarrow \llbracket a \rrbracket_{3, i}=0, \quad \llbracket c \rrbracket_{3, i}=1 ; \\
& \llbracket 2 x \rrbracket_{3, i} \neq 1 \Longrightarrow \llbracket a \rrbracket_{3, i}=\llbracket c \rrbracket_{3, i}=\frac{1}{2} \llbracket 2 x \rrbracket_{3, i} .
\end{aligned}
$$

We have $\llbracket a \rrbracket_{3, i} \leq \llbracket c \rrbracket_{3, i} \leq \llbracket 2 x \rrbracket_{3, i}$ for all $i \geq 0$. Also $\llbracket a \rrbracket_{3, j}=$ $0<\llbracket c \rrbracket_{3, j}=1$, because $\llbracket 2 x \rrbracket_{3, j}=1$. Hence $0 \leq a<c \leq 2 x$. It is easily checked that

$$
\llbracket a \rrbracket_{3, i}+\llbracket c \rrbracket_{3, i}=\llbracket 2 x \rrbracket_{3, i}
$$

for all $i \geq 0$, so $a+c=2 x$. Hence $x \in B_{0}^{+}$.

Next we show that $x$ is an upper endpoint for $\mathbb{Z}_{3}^{+}(0,1)$. Let $a, b \in \mathbb{Z}_{3}^{+}(0,1)$ be specified by their base 3 digits; thus

$$
\begin{gathered}
\llbracket x \rrbracket_{3, i}=2 \Longrightarrow \llbracket a \rrbracket_{3, i}=0, \quad \llbracket b \rrbracket_{3, i}=1 ; \\
\llbracket x \rrbracket_{3, i} \neq 2 \Longrightarrow \llbracket a \rrbracket_{3, i}=\llbracket b \rrbracket_{3, i}=\llbracket x \rrbracket_{3, i} .
\end{gathered}
$$

There is at least one $k \geq 0$ such that $\llbracket x \rrbracket_{3, k}=2$, so $0 \leq a<$ $b<x$. Also

$$
\llbracket a \rrbracket_{3, i}+\llbracket x \rrbracket_{3, i}=2 \llbracket b \rrbracket_{3, i}=\llbracket 2 b \rrbracket_{3, i}
$$

for all $i \geq 0$, so $a+x=2 b$. Hence $x \in C_{0}^{+}$.

Finally we show that $x$ is a lower endpoint for $\mathbb{Z}_{3}^{+}(0,1)$. We need to find $b, c \in \mathbb{Z}_{3}^{+}(0,1)$ such that $0<x<b<c$ and $x+c=2 b$. Base 3 arithmetic for this sum takes the form

$$
\llbracket x \rrbracket_{3, i}+\llbracket c \rrbracket_{3, i}+\delta_{i}=\llbracket 2 b \rrbracket_{3, i}+3 \delta_{i+1}
$$

for every $i \geq 0$, with carry-overs $\delta_{i} \in\{0,1\}$ satisfying $\delta_{0}=0$ and

$$
\begin{aligned}
& \llbracket x \rrbracket_{3, i}+\llbracket c \rrbracket_{3, i}+\delta_{i} \leq 2 \Longrightarrow \delta_{i+1}=0, \\
& \llbracket x \rrbracket_{3, i}+\llbracket c \rrbracket_{3, i}+\delta_{i}>2 \Longrightarrow \delta_{i+1}=1 .
\end{aligned}
$$

Specify $b, c$ by their base 3 digits; thus

$$
\begin{gathered}
\llbracket x \rrbracket_{3, i}+\delta_{i} \in\{0,3\} \Longrightarrow \llbracket c \rrbracket_{3, i}=\llbracket b \rrbracket_{3, i}=0, \\
\llbracket x \rrbracket_{3, i}+\delta_{i}=1 \Longrightarrow \llbracket c \rrbracket_{3, i}=\llbracket b \rrbracket_{3, i}=1, \\
\llbracket x \rrbracket_{3, i}+\delta_{i}=2 \Longrightarrow \llbracket c \rrbracket_{3, i}=1, \quad \llbracket b \rrbracket_{3, i}=0 .
\end{gathered}
$$

If $\llbracket x \rrbracket_{3, k}=2$ and $\llbracket x \rrbracket_{3, i}<2$ for $0 \leq i<k$ then $\delta_{i}=0$ for $0 \leq i \leq k$. It follows that $\llbracket c \rrbracket_{3, k}=1>\llbracket b \rrbracket_{3, k}=0$. Since $\llbracket c \rrbracket_{3, i} \geq \llbracket b \rrbracket_{3, i}$, for every $i \geq 0$, we have $c>b$.

There are integers $h \geq g \geq k$ such that $\llbracket x \rrbracket_{3, h}>0$ and $\llbracket x \rrbracket_{3, i}=0$ for $i>h$ and $\llbracket x \rrbracket_{3, g}=2$ and $\llbracket x \rrbracket_{3, i} \leq 1$ for $i>g$. If $\delta_{g}=0$ then $\llbracket c \rrbracket_{3, g}=1$, $\llbracket b \rrbracket_{3, g}=0$; if $\delta_{g}=1$ then $\llbracket c \rrbracket_{3, g}=\llbracket b \rrbracket_{3, g}=0$. In either case it follows that $\delta_{g+1}=1$. If $\delta_{h+1}=1$ then $\llbracket x \rrbracket_{3, h+1}<\llbracket c \rrbracket_{3, h+1}=\llbracket b \rrbracket_{3, h+1}=1$ so $x<b$ since $\llbracket x \rrbracket_{3, i}=0$ for all $i>h$. If $\delta_{h+1}=0$ then there is an integer $f$ such that $h \geq f>g$ and $\delta_{i}=1$ for $f \geq i>g$ while $\delta_{f+1}=0$. If $\llbracket x \rrbracket_{3, i} \leq 1$ and $\delta_{i}=0$ then $\llbracket x \rrbracket_{3, i}=\llbracket c \rrbracket_{3, i}=\llbracket b \rrbracket_{3, i} \in\{0,1\}$ so $\delta_{i+1}=0$. Hence $\delta_{i}=0$ for all $i>f$. Also $\delta_{f}=1, \delta_{f+1}=0$ and $\llbracket c \rrbracket_{3, f}=1$, so $\llbracket x \rrbracket_{3, f}=0$. Then $\llbracket x \rrbracket_{3, f}=0<\llbracket b \rrbracket_{3, f}=1$ and $\llbracket x \rrbracket_{3, i}=\llbracket b \rrbracket_{3, i}$ for all $i>f$, so $x<b$.

Finally, in all three cases $\llbracket x \rrbracket_{3, i}+\llbracket c \rrbracket_{3, i}+\delta_{i}=2 \llbracket b \rrbracket_{3, i}+3 \delta_{i+1}$ for each $i \geq 0$, so $x+c=2 b$. Hence $x \in A_{0}^{+}$.

Corollary 19. The greedy midpoint-free subset $\mathbb{Z}_{3}^{+}(0,1)$ is maximal in $\mathbb{Z}^{+}$.

In [3] it was shown that $S_{0}=\mathbb{Z}_{3}^{+}(0,1)$ is actually a maximal midpoint-free subset of $\mathbb{Z}$. The proof is not repeated here, but let us note that the single example $-32 \in A\left(S_{0}, \mathbb{Z}\right)$ follows from computing $-32+c=2 b$ with $b, c \in \mathbb{Z}_{3}^{+}(0,1)$ in the form $32+2 b=c$. Base 3 considerations yield

$$
(1012)_{3}+(22)_{3}=(1111)_{3} \text {, }
$$

corresponding to $(-32,4,40) \in \Lambda(\mathbb{Z})$, so $-32 \in A\left(S_{0}, \mathbb{Z}\right)$ as claimed.

For brevity, let $A_{0}:=A\left(S_{0}, \mathbb{Z}\right), B_{0}:=B\left(S_{0}, \mathbb{Z}\right), C_{0}:=$ $C\left(S_{0}, \mathbb{Z}\right)$. With Theorem 18 , this yields the following result.

Corollary 20. The greedy midpoint-free subset $\mathbb{Z}_{3}^{+}(0,1) \subset \mathbb{Z}$ has greedy balance point sets satisfying $A_{0}=\mathbb{Z} \backslash \mathbb{Z}_{3}^{+}(0,1)$, $B_{0}=C_{0}=\mathbb{Z}^{+} \backslash \mathbb{Z}_{3}^{+}(0,1)$.

Reversing implications, this yields the following result.

Corollary 21. The greedy midpoint-free subset $\mathbb{Z}_{3}^{+}(0,1)$ is maximal in $\mathbb{Z}$.

Now the multiplicities for $x \in \mathbb{Z}^{+} \backslash \mathbb{Z}_{3}^{+}(0,1)$ can be examined. First note that the sum of base 3 digits of any even integer is $0 \bmod 2$, so even integers have an even number of base 3 digits equal to 1 . If $x \in \mathbb{Z}^{+} \backslash \mathbb{Z}_{3}^{+}(0,1)$, at least one digit in $(2 x)_{3}$ must be 1 , so the total number of such digits is $2 k$, and $k>0$.

Theorem 22. If $x \in \mathbb{Z}^{+} \backslash \mathbb{Z}_{3}^{+}(0,1)$, then the midpoint multiplicity of $x$ is

$$
\beta\left(x, \mathbb{Z}_{3}^{+}(0,1)\right)=2^{2 k-1},
$$

where $2 k$ is the number of digits equal to 1 in $(2 x)_{3}$ and $k>0$.

Proof. Suppose $a, c \in \mathbb{Z}_{3}^{+}(0,1)$ satisfy $a<c$ and $a+c=2 x$. There is no carry-over in the base 3 arithmetic for the sum, so $\llbracket a \rrbracket_{3, i}+\llbracket c \rrbracket_{3, i}=\llbracket 2 x \rrbracket_{3, i}$, for all $i \geq 0$. If $\llbracket 2 x \rrbracket_{3, i} \in\{0,2\}$ then we must have

$$
\llbracket a \rrbracket_{3, i}=\llbracket c \rrbracket_{3, i}=\frac{1}{2} \llbracket 2 x \rrbracket_{3, i} .
$$

There is an integer $j>0$ such that $\llbracket 2 x \rrbracket_{3, j}=1$ and $\llbracket 2 x \rrbracket_{3, i} \epsilon$ $\{0,2\}$ for all $i>j$, so $a<c$ forces $\llbracket a \rrbracket_{3, j}=0<\llbracket c \rrbracket_{3, j}=1$. However, when $j>i \geq 0$ and $\llbracket 2 x \rrbracket_{3, i}=1$, the requirement $\llbracket a \rrbracket_{3, i}+\llbracket c \rrbracket_{3, i}=1$ is satisfied if $\left\{\llbracket a \rrbracket_{3, i}, \llbracket c \rrbracket_{3, i}\right\}=\{0,1\}$. Both possibilities are consistent with $a<c$, so there are $2^{2 k-1}$ solutions in total. 
To illustrate, when $x=50$ then $2 x=(10201)_{3}$ so $\Lambda\left(\mathbb{Z}_{3}^{+}(0,1), \mathbb{Z}^{+}\right)$has just two triples with 50 as midpoint: $(9,50,91),(10,50,90)$. In contrast, when $x=20$ then $2 x=(1111)_{3}$ so $\Lambda\left(\mathbb{Z}_{3}^{+}(0,1), \mathbb{Z}^{+}\right)$has eight triples with 20 as midpoint, ranging from $(0,20,40)$ to $(13,20,27)$, namely, $(a, 20,40-a)$ for all $a \in \mathbb{Z}_{3}^{+}(0,1) \cap[0,13]$.

Fix $x \in \mathbb{Z}^{+} \backslash \mathbb{Z}_{3}^{+}(0,1)$. The digits of the base 3 representation $(x)_{3}$ include at least one 2 . An ordered pair of integers $(j, k)$ with $0 \leq j<k$ is critical for $x$ if $\llbracket x \rrbracket_{3, j}=2$ and $\llbracket x \rrbracket_{3, k}<2$, with $\llbracket x \rrbracket_{3, i}>0$ when $j \leq i<k$. Any two ordered pairs $(j, k)$ and $\left(j^{\prime}, k^{\prime}\right)$ critical for $x$ are independent if $[j, k] \cap\left[j^{\prime}, k^{\prime}\right]=\emptyset$. Any set of ordered pairs critical for $x$ is independent if every two members are independent. Let Crit $(x)$ denote the family of all sets of independent ordered pairs critical for $x$, including the empty set.

Theorem 23. If $x \in \mathbb{Z}^{+} \backslash \mathbb{Z}_{3}^{+}(0,1)$, then $x$ has total endpoint multiplicity

$$
\alpha\left(x, \mathbb{Z}_{3}^{+}(0,1)\right)+\gamma\left(x, \mathbb{Z}_{3}^{+}(0,1)\right)=\# \operatorname{Crit}(x) .
$$

Proof. Given $x \in \mathbb{Z}^{+} \backslash \mathbb{Z}_{3}^{+}(0,1)$, we seek $y, b \in \mathbb{Z}_{3}^{+}(0,1)$ such that $x+y=2 b$. Any solution necessarily satisfies $x \neq y$. Base 3 arithmetic for this sum takes the form

$$
\llbracket x \rrbracket_{3, i}+\llbracket y \rrbracket_{3, i}+\delta_{i}=2 \llbracket b \rrbracket_{3, i}+3 \delta_{i+1}
$$

for every $i \geq 0$, with carry-overs $\delta_{i} \in\{0,1\}$ satisfying $\delta_{0}=0$ and

$$
\begin{aligned}
& \llbracket x \rrbracket_{3, i}+\llbracket y \rrbracket_{3, i}+\delta_{i} \leq 2 \Longrightarrow \delta_{i+1}=0, \\
& \llbracket x \rrbracket_{3, i}+\llbracket y \rrbracket_{3, i}+\delta_{i}>2 \Longrightarrow \delta_{i+1}=1 .
\end{aligned}
$$

Specifying $y, b$ by their base 3 digits, the equation $x+y=2 b$ requires

$$
\begin{gathered}
\llbracket x \rrbracket_{3, i}+\delta_{i} \in\{0,3\} \Longrightarrow \llbracket y \rrbracket_{3, i}=\llbracket b \rrbracket_{3, i}=0, \\
\llbracket x \rrbracket_{3, i}+\delta_{i}=1 \Longrightarrow \llbracket y \rrbracket_{3, i}=\llbracket b \rrbracket_{3, i}=1, \\
\llbracket x \rrbracket_{3, i}+\delta_{i}=2 \Longrightarrow \llbracket y \rrbracket_{3, i}+\llbracket b \rrbracket_{3, i}=1 .
\end{gathered}
$$

As is easily verified, these digit specifications satisfy the requirements of base 3 arithmetic for $x+y=2 b$.

Any integer $i \geq 0$ is a base 3 transition point for the sum $x+y$ if $\delta_{i+1} \neq \delta_{i}$. Note that the digits $\llbracket y \rrbracket_{3, i}$ and $\llbracket b \rrbracket_{3, i}$ are forced unless $\llbracket x \rrbracket_{3, i}+\delta_{i}=2$. In the latter case there are two options:

(1) $\llbracket y \rrbracket_{3, i}=0, \llbracket b \rrbracket_{3, i}=1, \delta_{i+1}=0$,

(2) $\llbracket y \rrbracket_{3, i}=1, \llbracket b \rrbracket_{3, i}=0, \delta_{i+1}=1$.

If $\llbracket x \rrbracket_{3, i}=2, \delta_{i}=0$ then choosing option (1) preserves the carry-over state ("off") with $\delta_{i+1}=0$ whereas choosing option (2) switches the carry-over state (to "on") with $\delta_{i+1}=1$, so option (2) causes $i$ to be a transition point. If $\llbracket x \rrbracket_{3, i}=1$, $\delta_{i}=1$ then choosing option (1) switches the carry-over state (to "off") with $\delta_{i+1}=0$ and causes $i$ to be a transition point, whereas choosing option (2) preserves the carry-over state (“on") with $\delta_{i+1}=1$.
Let $X$ be a maximal block of nonzero digits in the base 3 representation $(x)_{3}$, say $X:=\left(\llbracket x \rrbracket_{3, i} \mid g \leq i \leq h\right)$, and assume that $X$ contains the digit 2 at least once. There must be at least one such maximal block $X$ in $(x)_{3}$. Then $\delta_{g}=0$, and the "off" carry-over state $\delta_{i}=0$ can only switch to $\delta_{j+1}=1$ for some $j$ such that $g \leq j \leq h$ when $\llbracket x \rrbracket_{3, j}=2$ and $\llbracket y \rrbracket_{3, j}=1$, corresponding to an option (2) choice in the construction of $y$. The "on" carry-over state $\delta_{i}=1$ can only switch back to $\delta_{k+1}=0$ for some $k$ such that $j<k \leq h$ if $\llbracket x \rrbracket_{3, k}=1$ and $\llbracket y \rrbracket_{3, k}=0$, corresponding to an option (1) choice in constructing $y$. If no such option is exercised, then maximality of $X$ ensures the carry-over state $\delta_{i}=1$ inevitably switches back at $i=h+1$, for in this case $\llbracket x \rrbracket_{3, h}>0$, $\llbracket x \rrbracket_{3, h+1}=0, \delta_{h}=1$ and an option (2) choice for $\llbracket y \rrbracket_{3, h}$ results in $\delta_{h+1}=1, \llbracket y \rrbracket_{3, h+1}=1, \llbracket b \rrbracket_{3, h+1}=1, \delta_{h+2}=0$. Note that the ordered pair $(j, k)$ is critical for $x$, as is the ordered pair $(j, h+1)$.

Now consider any set $P(X)$ of independent ordered pairs $(j, k)$ critical for $x$, with $g \leq j<k \leq h+1$. The set $P(X)$ determines a unique sequence of carry-over digits $\Delta:=\left(\delta_{i} \mid g \leq i \leq h+1\right)$, with $\delta_{i}=1$ if $j \leq i \leq k$ and $(j, k) \in P(X)$, and $\delta_{i}=0$ for every other $i$ in the interval $[g, h+1]$. Then $X$ and $\Delta$ determine blocks

$$
\begin{aligned}
Y & :=\left(\llbracket y \rrbracket_{3, i} \mid g \leq i \leq h+1\right), \\
B & :=\left(\llbracket b \rrbracket_{3, i} \mid g \leq i \leq h+1\right)
\end{aligned}
$$

such that $X+Y=2 B$. Each member of $\operatorname{Crit}(x)$ is of the form $P(x):=\bigcup_{X} P(X)$, where $X$ runs through all maximal blocks of nonzero digits containing 2 in $(x)_{3}$. Any such $P(x)$ uniquely determines a collection of suitable blocks of base 3 digits for $y, b$ while all other digits are forced by base 3 arithmetic for $x+y=2 b$, so the number of solutions for $y, b$ is precisely \#Crit $(x)$.

To illustrate, when $x=50=(1212)_{3}$ the possible ordered pairs critical for $x$ are $(0,1),(0,3),(0,4),(2,3),(2,4)$. There are \#Crit $(50)=8$ sets of independent critical pairs, namely, $\emptyset$, the five singletons, $\{(0,1),(2,3)\}$ and $\{(0,1),(2,4)\}$. The corresponding triples in $\Lambda\left(\mathbb{Z}^{+}\right)$with 50 as an endpoint are

$(4,27,50),(10,30,50),(12,31,50),(28,39,50),(30,40,50)$,

so $\gamma\left(50, \mathbb{Z}_{3}^{+}(0,1)\right)=5$;

$(50,81,112),(50,84,118),(50,85,120)$,

so $\alpha\left(50, \mathbb{Z}_{3}^{+}(0,1)\right)=3$.

Given $x \in \mathbb{Z}^{+} \backslash \mathbb{Z}_{3}^{+}(0,1)$, let $X:=\left(\llbracket x \rrbracket_{3, i} \mid g \leq i \leq h\right)$ be the leading maximal block of nonzero digits containing 2 in $(x)_{3}$, so $\llbracket x \rrbracket_{3, i}<2$ when $i>h$, and in particular $\llbracket x \rrbracket_{3, h+1}=0$. Then any ordered pair $(j, k)$ critical for $x$ is constrained by $0 \leq j<k \leq h+1$. In particular, the leading ordered pairs critical for $x$ are all those of the form $(j, h+1)$. In this case $g \leq j \leq h$, and there is at least one such ordered pair since $\llbracket x \rrbracket_{3, j}=2$ for at least one $j \in[g, h]$. Let LeadCrit $(x)$ denote the family of just those sets of independent ordered pairs critical for $x$ that include a leading pair. 
Corollary 24. If $x \in \mathbb{Z}^{+} \backslash \mathbb{Z}_{3}^{+}(0,1)$, then $x$ has lower endpoint multiplicity

$$
\alpha\left(x, \mathbb{Z}_{3}^{+}(0,1)\right)=\text { \#LeadCrit }(x) .
$$

Proof. Continuing with the definitions and notation in the proof of Theorem 23, if $P(x) \in \operatorname{LeadCrit}(x)$ contains the leading ordered pair $(j, h+1)$ critical for $x$ then the corresponding $y, b$ for $x+y=2 b$ satisfy $\llbracket x \rrbracket_{3, j}=2, \llbracket y \rrbracket_{3, j}=1, \llbracket b \rrbracket_{3, j}=0$ with $\llbracket y \rrbracket_{3, i}=\llbracket b \rrbracket_{3, i}$ when $i>j$; moreover, $\llbracket x \rrbracket_{3, h+1}=0$, $\llbracket y \rrbracket_{3, h+1}=1$, and $\llbracket x \rrbracket_{3, i}=\llbracket y \rrbracket_{3, i}$ when $i \geq h+2$. Thus $x<b<y$ and $b, y \in \operatorname{supp}_{A}\left(x, \mathbb{Z}_{3}^{+}(0,1)\right)$.

On the other hand, if $P^{*}(x) \in \operatorname{Crit}(x) \backslash \operatorname{LeadCrit}(x)$ then the corresponding $y, b$ for $x+y=2 b$ satisfy $\llbracket x \rrbracket_{3, k}+\delta_{k}=2$, $\llbracket y \rrbracket_{3, k}=0, \llbracket b \rrbracket_{3, k}=1$ for some $k \in[g, h]$, with $\llbracket x \rrbracket_{3, i}=$ $\llbracket y \rrbracket_{3, k}=\llbracket b \rrbracket_{3, i}$ when $i>k$. Thus $b, y \in \operatorname{supp}_{C}\left(x, \mathbb{Z}_{3}^{+}(0,1)\right)$.

The multiplicities in Theorem 23 and Corollary 24 are implicit, so it is of some interest to identify a class of positive integers with endpoint multiplicities that can be specified simply and explicitly. Let $u_{3, n}:=\left(3^{n}-1\right) / 2$ be the integer with base 3 representation comprising a block of $n$ digits all equal to 1 , so $u_{3, n}$ is a base 3 rep-unit, following terminology of Yates [6]. (See A003462 in OEIS [5].)

Corollary 25. For any integers $n \geq m>0$ and $s \geq 0$, $t>n+s$, let

$$
x:=3^{s}\left(u_{3, n}+u_{3, m}\right)+3^{t} v+w,
$$

where $v \in \mathbb{Z}_{3}^{+}(0,1)$ and $w \in \mathbb{Z}_{3}^{+}(0,1) \cap\left[0,3^{s}\right)$. Then

$$
\begin{gathered}
\alpha\left(x, \mathbb{Z}_{3}^{+}(0,1)\right)=m, \\
\gamma\left(x, \mathbb{Z}_{3}^{+}(0,1)\right)=m(n-m)+1 .
\end{gathered}
$$

Proof. The only maximal block of nonzero digits containing 2 in $(x)_{3}$ is $X=\left(\llbracket x \rrbracket_{3, i} \mid s \leq i<n+s\right)$, with $\llbracket x \rrbracket_{3, i}=2$ when $s \leq i<m+s$, and $\llbracket x \rrbracket_{3, i}=1$ when $m+s \leq i<n+s$, with $\llbracket x \rrbracket_{3, n+s}=0$. Therefore, the only ordered pairs $(j, k)$ critical for $x$ have $s \leq j<m+s \leq k \leq n+s$. No two of these critical pairs are disjoint, so the only sets in Crit $(x)$ are $m(n-m+1)$ singletons and the empty set. The sets comprising LeadCrit $(x)$ are the $m$ singletons $\{(j, n+s)\}$.

For instance, $x=862=(1011221)_{3}$ arises by taking $m=$ $2, n=4, s=1, t=6$, and $v=w=1$ in Corollary 25. The leading critical pairs for $x$ are $(j, 5)$ with $j \in\{1,2\}$, yielding two midpoint triples $(x, b, y)$ with $b, y \in \mathbb{Z}_{3}^{+}(0,1)$ :

$$
b=(11000 B 1)_{3}, \quad y=(1111 Y 1)_{3}
$$

for blocks $(B, Y) \in\{(0,01),(1,10)\}$, so $(x, b, y)=(862$, $973+r, 1084+2 r)$ with $r \in\{0,3\}$. Thus $\alpha\left(862, \mathbb{Z}_{3}^{+}(0,1)\right)=$ 2. The non-leading critical pairs for $x$ are $(j, k)$ with $j \in$ $\{1,2\}, k \in\{3,4\}$, yielding five midpoint triples $(y, b, x)$ with $b, y \in \mathbb{Z}_{3}^{+}(0,1)$ :

$$
b=(101 B 1)_{3}, \quad y=(10 Y 1)_{3}
$$

for $(B, Y) \in\{(000,0101),(001,0110),(100,1001),(101,1010)$, $(111,1100)\}$, so $(y, b, x)=(760+2 r, 811+r, 862)$ for $r \in\{0,3,27,30,39\}$. Thus $\gamma\left(862, \mathbb{Z}_{3}^{+}(0,1)\right)=5$.

\section{Doubling the Greedy Midpoint-Free Subset of $\mathbb{Z}^{+}$}

Doubling $S_{0}:=\mathbb{Z}_{3}^{+}(0,1)$ as in Theorem 4 shows that $S_{0}^{(2)}:=2 S_{0} \cup-\left(2 S_{0}+1\right)$ is a maximal midpoint-free subset of $\mathbb{Z}$. (This is an alternative demonstration to the proof given in [3].) Here $2 S_{0}=\mathbb{Z}_{3}^{+}(0,2)$ and $2 S_{0}+1=\mathbb{Z}_{3}^{+}(0,2 ; 1)$, where $\mathbb{Z}_{3}^{+}(0,2 ; 1)$ comprises those positive integers with base 3 representation in which the trailing digit (the last nonzero digit) is 1 and every other digit is in $\{0,2\}$. The relevant balance point sets and multiplicities will now be examined briefly.

For brevity, let us write

$$
\begin{aligned}
& A_{0,2}^{+}:=A\left(2 S_{0}, \mathbb{Z}^{+}\right), \quad B_{0,2}^{+}:=B\left(2 S_{0}, \mathbb{Z}^{+}\right), \\
& C_{0,2}^{+}:=C\left(2 S_{0}, \mathbb{Z}^{+}\right) ; \\
& A_{0,2}:=A\left(2 S_{0}, \mathbb{Z}\right), \quad B_{0,2}:=B\left(2 S_{0}, \mathbb{Z}\right), \\
& C_{0,2}:=C\left(2 S_{0}, \mathbb{Z}\right) ; \\
& A_{0,2}^{(2)}:=A\left(S_{0}^{(2)}, \mathbb{Z}\right), \quad B_{0,2}^{(2)}:=B\left(S_{0}^{(2)}, \mathbb{Z}\right), \\
& C_{0,2}^{(2)}:=C\left(S_{0}^{(2)}, \mathbb{Z}\right) .
\end{aligned}
$$

Note that $A\left(2 S_{0}+1, \mathbb{Z}^{+}\right)=A_{0,2}^{+}+1, A\left(2 S_{0}+1, \mathbb{Z}\right)=A_{0,2}+1$. Similar identities hold for other balance point sets of $2 S_{0}+1$.

It can be shown that every odd integer $x \in \mathbb{Z}^{+}$is the sum of two distinct members of $\mathbb{Z}_{3}^{+}(0,1)$. Indeed, there are precisely $4^{k}$ such sums, where $2 k+1$ is the number of digits equal to 1 in $(x)_{3}$ for some integer $k \geq 0$. The balance point sets and multiplicities for $2 S_{0}, 2 S_{0}+1$, and $S_{0}^{(2)}$ can now be specified.

Theorem 26. The midpoint-free subset $\mathbb{Z}_{3}^{+}(0,2) \subset \mathbb{Z}^{+}$has balance point sets satisfying $A_{0,2}^{+}=C_{0,2}^{+}=2 \mathbb{Z}^{+} \backslash \mathbb{Z}_{3}^{+}(0,2)$ and $B_{0,2}^{+}=\mathbb{Z}^{+} \backslash \mathbb{Z}_{3}^{+}(0,2)$.

Corollary 27. If $x \in \mathbb{Z}^{+} \backslash \mathbb{Z}_{3}^{+}(0,1)$, the endpoint multiplicities satisfy

$$
\begin{aligned}
& \alpha\left(2 x+1, \mathbb{Z}_{3}^{+}(0,2 ; 1)\right)=\alpha\left(2 x, \mathbb{Z}_{3}^{+}(0,2)\right)=\alpha\left(x, \mathbb{Z}_{3}^{+}(0,1)\right), \\
& \gamma\left(2 x+1, \mathbb{Z}_{3}^{+}(0,2 ; 1)\right)=\gamma\left(2 x, \mathbb{Z}_{3}^{+}(0,2)\right)=\gamma\left(x, \mathbb{Z}_{3}^{+}(0,1)\right) .
\end{aligned}
$$

Corollary 28. If $x \in \mathbb{Z}^{+} \backslash \mathbb{Z}_{3}^{+}(0,2)$, the midpoint multiplicities satisfy

$$
\beta\left(x+1, \mathbb{Z}_{3}^{+}(0,2 ; 1)\right)=\beta\left(x, \mathbb{Z}_{3}^{+}(0,2)\right)=2^{k-1},
$$

where $k>0$ is the number of digits equal to 1 in $(x)_{3}$.

Corollary 29. The midpoint-free subset $\mathbb{Z}_{3}^{+}(0,2) \subset \mathbb{Z}$ has balance point sets satisfying $A_{0,2}=A_{0,2}^{+} \cup 2 \mathbb{Z}^{-}, B_{0,2}=B_{0,2}^{+}$, $C_{0,2}=C_{0,2}^{+}$. 
Corollary 30. The midpoint-free subset $S_{0}^{(2)}:=\mathbb{Z}_{3}^{+}(0,2) \cup$ $-\mathbb{Z}_{3}^{+}(0,2 ; 1) \subset \mathbb{Z}$ has balance point sets satisfying

$$
\begin{gathered}
A_{0,2}^{(2)}=A_{0,2} \cup-\left(C_{0,2}+1\right)=\left(\mathbb{Z} \backslash S_{0}^{(2)}\right) \backslash\left(2 \mathbb{Z}^{+}+1\right), \\
C_{0,2}^{(2)}=C_{0,2} \cup-\left(A_{0,2}+1\right)=\left(\mathbb{Z} \backslash S_{0}^{(2)}\right) \backslash 2 \mathbb{Z}^{-}, \\
B_{0,2}^{(2)}=B_{0,2}^{+} \cup-B_{0,2,1}^{+}=\mathbb{Z} \backslash S_{0}^{(2)} .
\end{gathered}
$$

Corollary 31. The set $S_{0}^{(2)}$ is a maximal midpoint-free subset of $\mathbb{Z}$, with balance point sets satisfying $A_{0,2}^{(2)} \cup C_{0,2}^{(2)}=B_{0,2}^{(2)}=\mathbb{Z} \backslash S_{0}^{(2)}$.

Note that trebling $S_{0}:=\mathbb{Z}_{3}^{+}(0,1)$ as in Theorem 6 yields $S_{0}^{(3)}:=3 S_{0} \cup\left(3 S_{0}+1\right)$, and earlier we saw that this is "trivial" in this case, because $S_{0}^{(3)}=S_{0}$. In the notation of Corollary 7, for any integer $n \geq 1$ this implies

$$
S_{0}^{(3, n)}=S_{0}, \quad S_{0}^{(2,3, n)}=S_{0}^{(2)} .
$$

If $S$ is any set satisfying the hypotheses of Theorem 6, and $s_{0}:=\min (S)$, then the normalized set $S^{\prime}=S-s_{0}$ is an affine transform which satisfies the hypotheses of Theorem 6. Without loss of generality, assume $S$ is any normalized compliant set; then Corollary 7 shows that iterated trebling yields a sequence of maximal midpoint-free subsets of $\mathbb{Z}$ which asymptotically approach the greedy subset $S_{0}$, because

$$
S^{(3, n)} \cap\left[0,3^{n}\right)=S_{0} \cap\left[0,3^{n}\right) .
$$

In this sense we may write the asymptotic equivalences

$$
S^{(3, n)} \sim S_{0}, \quad S^{(2,3, n)} \sim S_{0}^{(2)} .
$$

\section{The Midpoint-Free Set $\mathbb{Z}_{4}^{+}(0,1)$}

Let us now study the set

$$
S_{1}:=\mathbb{Z}_{4}^{+}(0,1)=\{0,1,4,5,16,17,20,21,64, \ldots\},
$$

corresponding to sequence A000695 in OEIS [5]. Corollaries 9 and 15 leave open the possibility that $S_{1}$ is a maximal midpoint-free subset of $\mathbb{Z}^{+}$. We now settle that matter. Let $A_{1}^{+}:=A\left(S_{1}, \mathbb{Z}^{+}\right), B_{1}^{+}:=B\left(S_{1}, \mathbb{Z}^{+}\right), C_{1}^{+}:=C\left(S_{1}, \mathbb{Z}^{+}\right)$.

Theorem 32. The midpoint-free subset $\mathbb{Z}_{4}^{+}(0,1) \subset \mathbb{Z}^{+}$has endpoint sets satisfying $A_{1}^{+} \cup C_{1}^{+}=\mathbb{Z}^{+} \backslash \mathbb{Z}_{4}^{+}(0,1)$.

Proof. Let $x \in \mathbb{Z}^{+} \backslash \mathbb{Z}_{4}^{+}(0,1)$. We seek $y, b \in \mathbb{Z}_{4}^{+}(0,1)$ such that $x+y=2 b$. Clearly any such solution has $x \neq y$. Base 4 computation requires

$$
\llbracket x \rrbracket_{4, i}+\llbracket y \rrbracket_{4, i}+\delta_{i}=2 \llbracket b \rrbracket_{4, i}+4 \delta_{i+1}
$$

for every $i \geq 0$, with carry-overs $\delta_{i} \in\{0,1\}$ satisfying $\delta_{0}=0$ and

$$
\begin{aligned}
& \llbracket x \rrbracket_{4, i}+\llbracket y \rrbracket_{4, i}+\delta_{i} \leq 3 \Longrightarrow \delta_{i+1}=0, \\
& \llbracket x \rrbracket_{4, i}+\llbracket y \rrbracket_{4, i}+\delta_{i}>3 \Longrightarrow \delta_{i+1}=1 .
\end{aligned}
$$

Specifying $y, b$ by their base 4 digits, the equation $x+y=2 b$ requires

$$
\begin{gathered}
\llbracket x \rrbracket_{4, i}+\delta_{i} \in\{0,4\} \Longrightarrow \llbracket y \rrbracket_{4, i}=\llbracket b \rrbracket_{4, i}=0, \\
\llbracket x \rrbracket_{4, i}+\delta_{i}=1 \Longrightarrow \llbracket y \rrbracket_{4, i}=\llbracket b \rrbracket_{4, i}=1, \\
\llbracket x \rrbracket_{4, i}+\delta_{i}=2 \Longrightarrow \llbracket y \rrbracket_{4, i}=0, \quad \llbracket b \rrbracket_{4, i}=1, \\
\llbracket x \rrbracket_{4, i}+\delta_{i}=3 \Longrightarrow \llbracket y \rrbracket_{4, i}=1, \quad \llbracket b \rrbracket_{4, i}=0 .
\end{gathered}
$$

These digit specifications are easily seen to satisfy the requirements of base 4 arithmetic for $x+y=2 b$. Therefore, every $x \in \mathbb{Z}^{+} \backslash \mathbb{Z}_{4}^{+}(0,1)$ is in $A_{1}^{+} \cup C_{1}^{+}$. Since $\mathbb{Z}_{4}^{+}(0,1)$ is midpointfree, it follows that $A_{1}^{+} \cup C_{1}^{+}=\mathbb{Z}^{+} \backslash \mathbb{Z}_{4}^{+}(0,1)$.

Corollary 33. The set $\mathbb{Z}_{4}^{+}(0,1)$ is a maximal midpoint-free subset of $\mathbb{Z}^{+}$.

Corollary 34. For any $x \in \mathbb{Z}^{+} \backslash \mathbb{Z}_{4}^{+}(0,1)$ the total endpoint multiplicity is

$$
\alpha\left(x, \mathbb{Z}_{4}^{+}(0,1)\right)+\gamma\left(x, \mathbb{Z}_{4}^{+}(0,1)\right)=1 .
$$

Any $x \in \mathbb{Z}^{+} \backslash \mathbb{Z}_{4}^{+}(0,1)$ has at least one base 4 digit in $\{2,3\}$. A high block in $(x)_{4}$ is a maximal block of base 4 digits all in $\{2,3\}$. If $X:=\left(\llbracket x \rrbracket_{4, i} \mid j \leq i \leq k\right)$ is a high block in $(x)_{4}$, it is clear if $\llbracket x \rrbracket_{4, k+1}=0$ and leading if $\llbracket x \rrbracket_{4, i} \leq 1$ when $i>k$.

Corollary 35. The lower endpoint set $A_{1}^{+}$for $\mathbb{Z}_{4}^{+}(0,1)$ in $\mathbb{Z}^{+}$ comprises all positive integers in which the leading base 4 high block is clear and contains 3 . The upper endpoint set $C_{1}^{+}$is the complement of $A_{1}^{+}$in $\mathbb{Z}^{+} \backslash \mathbb{Z}_{4}^{+}(0,1)$.

Proof. With the definitions and notation in the proof of Theorem 32, let $\llbracket x \rrbracket_{4, h}>0$ for some $h>0$ and $\llbracket x \rrbracket_{4, i}=0$ when $i>h$. Let $X:=\left(\llbracket x \rrbracket_{4, i} \mid j \leq i \leq k\right)$ be the leading base 4 high block for $x$, so $0 \leq j \leq k \leq h$ and $\llbracket x \rrbracket_{4, i} \leq 1$ when $i>k$.

(1) If $\llbracket x \rrbracket_{4, h}=1$ then $\delta_{h+1}=0$, so $\llbracket y \rrbracket_{4, i}=\llbracket b \rrbracket_{4, i}=0$ when $i>h$. Also $\llbracket x \rrbracket_{4, k} \geq 2$, while $\llbracket x \rrbracket_{4, i} \leq 1$ when $i>k$. If $\llbracket x \rrbracket_{4, k}+\delta_{k} \geq 3$ then $\delta_{k+1}=1$. Therefore, $\llbracket x \rrbracket_{4, k+1}+\llbracket y \rrbracket_{4, k+1}=1$ and $\delta_{i}=0$ when $i>k+1$. This case arises just when $\llbracket x \rrbracket_{4, t}=3$ for some $t$ such that $j \leq t \leq k$, and $\llbracket x \rrbracket_{4, i}=2$ if $t<i \leq k$. Also $\llbracket x \rrbracket_{4, i}=$ $\llbracket y \rrbracket_{4, i}$ when $i>k+1$, so $x>y$ if $\llbracket x \rrbracket_{4, k+1}=1$ and $x<y$ if $\llbracket x \rrbracket_{4, k+1}=0$, the latter condition holding precisely when $X$ is clear. On the other hand, if $\llbracket x \rrbracket_{4, k}+\delta_{k}=2$ then $\llbracket x \rrbracket_{4, k}=2, \llbracket y \rrbracket_{4, k}=0$, while $\delta_{i}=0$ and $\llbracket x \rrbracket_{4, i}=\llbracket y \rrbracket_{4, i}$ when $i>k$, so $x>y$.

(2) If $\llbracket x \rrbracket_{4, h}=2$ and $\delta_{h}=0$ then $\llbracket y \rrbracket_{4, h}=0$, so $\delta_{i}=0$ and $\llbracket x \rrbracket_{4, i}=\llbracket y \rrbracket_{4, i}=0$ when $i>h$. Then $k=h$ but the high block $X$ does not contain 3. In this case $x>y$.

(3) If $\llbracket x \rrbracket_{4, h}+\delta_{h}=3$ then $\llbracket y \rrbracket_{4, h}=1$, so $\delta_{h+1}=1$, $\llbracket x \rrbracket_{4, h+1}=0, \llbracket y \rrbracket_{4, h+1}=1$, and $\delta_{i}=\llbracket x \rrbracket_{4, i}=$ $\llbracket y \rrbracket_{4, i}=0$ when $i>h+1$. Once again $k=h$, but now the high block $X$ must contain 3. In this case $x<y$.

(4) If $\llbracket x \rrbracket_{4, h}=3, \delta_{h}=1$ then $\delta_{h+1}=1, \llbracket x \rrbracket_{4, h+1}=0$, $\llbracket y \rrbracket_{4, h+1}=1$ and the same conclusions as in (3) apply. 
Corollary 36. The midpoint set $B_{1}^{+}$for $\mathbb{Z}_{4}^{+}(0,1)$ in $\mathbb{Z}^{+}$comprises those positive integers $x$ with $2 x \in \mathbb{Z}_{4}^{+}(0,1,2) \backslash \mathbb{Z}_{4}^{+}(0,2)$. Any such $x$ has midpoint multiplicity $\beta\left(x, \mathbb{Z}_{4}^{+}(0,1)\right)=2^{k-1}$, where $k>0$ is the number of digits in $(2 x)_{4}$ equal to 1 .

Proof. Let $x \in \mathbb{Z}^{+} \backslash \mathbb{Z}_{4}^{+}(0,1)$. We seek $a, c \in \mathbb{Z}_{4}^{+}(0,1)$ such that $a+c=2 x$. Clearly any such solution has $a \neq x$, so $a \neq c$ holds. Base 4 arithmetic requires

$$
\llbracket a \rrbracket_{4, i}+\llbracket c \rrbracket_{4, i}=\llbracket 2 x \rrbracket_{4, i}
$$

for every $i \geq 0$. This forces $\llbracket 2 x \rrbracket_{4, i} \in\{0,1,2\}$. Since $x \notin$ $\mathbb{Z}_{4}^{+}(0,1)$ it follows that $\llbracket 2 x \rrbracket_{4, j}=1$ for some $j \geq 0$, with $\llbracket 2 x \rrbracket_{4, i} \neq 1$ for all $i>j$. Specifying $a, c$ by their base 4 digits, the equation $a+c=2 x$ requires

$$
\begin{gathered}
\llbracket 2 x \rrbracket_{4, i} \in\{0,2\} \Longrightarrow \llbracket a \rrbracket_{4, i}=\llbracket c \rrbracket_{4, i}=\frac{1}{2} \llbracket 2 x \rrbracket_{4, i}, \\
\llbracket 2 x \rrbracket_{4, i}=1 \Longrightarrow \llbracket a \rrbracket_{4, i}+\llbracket c \rrbracket_{4, i}=1 .
\end{gathered}
$$

With $\llbracket a \rrbracket_{4, j}=0, \llbracket c \rrbracket_{4, j}=1$, clearly all solutions meet base 4 arithmetic requirements for $a+c=2 x$ and $a<c$.

An alternative characterization of the midpoint set $B_{1}^{+}$is that it comprises every $x \in \mathbb{Z}^{+}$with $\llbracket x \rrbracket_{4, i} \in\{2,3\}$ for at least one $i \geq 0$, and in each such instance $\llbracket x \rrbracket_{4, i+1} \in\{0,2\}$.

Now consider the midpoint-free set $S_{1}:=\mathbb{Z}_{4}^{+}(0,1)$ as a subset of $\mathbb{Z}$. For brevity, let $A_{1}:=A\left(S_{1}, \mathbb{Z}\right), B_{1}:=B\left(S_{1}, \mathbb{Z}\right)$, $C_{1}:=C\left(S_{1}, \mathbb{Z}\right)$.

Corollary 37. The midpoint-free subset $\mathbb{Z}_{4}^{+}(0,1) \subset \mathbb{Z}$ has balance point sets satisfying $A_{1}=A_{1}^{+} \cup \mathbb{Z}^{-}, B_{1}=B_{1}^{+}, C_{1}=C_{1}^{+}$.

Proof. Let $-x \in \mathbb{Z}^{-}$. We seek $b, c \in \mathbb{Z}_{4}^{+}(0,1)$ such that $x+2 b=c$. Clearly any such solution has $-x<b<c$. Base 4 computation requires

$$
\llbracket x \rrbracket_{4, i}+2 \llbracket b \rrbracket_{4, i}+\delta_{i}=\llbracket c \rrbracket_{4, i}+4 \delta_{i+1}
$$

for every $i \geq 0$, with carry-overs $\delta_{i} \in\{0,1\}$ satisfying $\delta_{0}=0$ and

$$
\begin{aligned}
& \llbracket x \rrbracket_{4, i}+2 \llbracket b \rrbracket_{4, i}+\delta_{i} \leq 3 \Longrightarrow \delta_{i+1}=0, \\
& \llbracket x \rrbracket_{4, i}+2 \llbracket b \rrbracket_{4, i}+\delta_{i}>3 \Longrightarrow \delta_{i+1}=1 .
\end{aligned}
$$

Specifying $b, c$ by their base 4 digits, we require

$$
\begin{gathered}
\llbracket x \rrbracket_{4, i}+\delta_{i} \in\{0,4\} \Longrightarrow \llbracket b \rrbracket_{4, i}=\llbracket c \rrbracket_{4, i}=0, \\
\llbracket x \rrbracket_{4, i}+\delta_{i}=1 \Longrightarrow \llbracket b \rrbracket_{4, i}=0, \quad \llbracket c \rrbracket_{4, i}=1, \\
\llbracket x \rrbracket_{4, i}+\delta_{i}=2 \Longrightarrow \llbracket b \rrbracket_{4, i}=1, \quad \llbracket c c \rrbracket_{4, i}=0, \\
\llbracket x \rrbracket_{4, i}+\delta_{i}=3 \Longrightarrow \llbracket b \rrbracket_{4, i}=\llbracket c \rrbracket_{4, i}=1 .
\end{gathered}
$$

It is straightforward to verify that $b, c$ are uniquely determined by these specifications, and $(-x, b, c) \in \Lambda(\mathbb{Z})$, so $-x \in A_{1}$.

Corollary 38. The set $\mathbb{Z}_{4}^{+}(0,1)$ is a maximal midpoint-free subset of $\mathbb{Z}$.
Corollary 39. Any $x \in \mathbb{Z}^{-}$has lower endpoint multiplicity $\alpha\left(x, \mathbb{Z}_{4}^{+}(0,1)\right)=1$.

\section{Doubling and Trebling the Set $\mathbb{Z}_{4}^{+}(0,1)$}

By Theorem 32 and Corollary 38, the set $S_{1}:=\mathbb{Z}_{4}^{+}(0,1)$ is compliant with the requirements for the doubling and trebling constructions of Theorems 4 and 6 , so both sets

$$
S_{1}^{(2)}:=2 S_{1} \cup-\left(2 S_{1}+1\right), \quad S_{1}^{(3)}:=3 S_{1} \cup\left(3 S_{1}+1\right)
$$

are maximal midpoint-free subsets of $\mathbb{Z}$.

Let us now briefly examine the balance point sets associated with $S_{1}^{(2)}$ :

$$
\begin{aligned}
& A_{1,2}^{+}:=A\left(2 S_{1}, \mathbb{Z}^{+}\right), \quad B_{1,2}^{+}:=B\left(2 S_{1}, \mathbb{Z}^{+}\right), \\
& C_{1,2}^{+}:=C\left(2 S_{1}, \mathbb{Z}^{+}\right) ; \\
& A_{1,2}:=A\left(2 S_{1}, \mathbb{Z}\right), \quad B_{1,2}:=B\left(2 S_{1}, \mathbb{Z}\right), \\
& C_{1,2}:=C\left(2 S_{1}, \mathbb{Z}\right) ; \\
& A_{1,2}^{(2)}:=A\left(S_{1}^{(2)}, \mathbb{Z}\right), \quad B_{1,2}^{(2)}:=B\left(S_{1}^{(2)}, \mathbb{Z}\right), \\
& C_{1,2}^{(2)}:=C\left(S_{1}^{*}, \mathbb{Z}\right) .
\end{aligned}
$$

Easy digit and parity considerations in combination with Theorem 32 yield the following corollaries.

Theorem 40. The midpoint-free subset $2 S_{1}=\mathbb{Z}_{4}^{+}(0,2) \subset \mathbb{Z}^{+}$ has balance point sets satisfying

$$
\begin{aligned}
& A_{1,2}^{+}=2 A_{1}^{+}, \quad C_{1,2}^{+}=2 C_{1}^{+}, \\
& B_{1,2}^{+}=\mathbb{Z}_{4}^{+}(0,1,2) \backslash \mathbb{Z}_{4}^{+}(0,2) .
\end{aligned}
$$

Corollary 41. The midpoint-free subset $2 S_{1} \subset \mathbb{Z}$ has balance point sets satisfying

$$
A_{1,2}=A_{1,2}^{+} \cup 2 \mathbb{Z}^{-}, \quad B_{1,2}=B_{1,2}^{+}, \quad C_{1,2}=C_{1,2}^{+} .
$$

Corollary 42. The subset $S_{1}^{(2)}:=2 S_{1} \cup-\left(2 S_{1}+1\right) \subset \mathbb{Z}$ is midpoint-free and has balance point sets satisfying

$$
\begin{aligned}
& A_{1,2}^{(2)}=A_{1,2} \cup-\left(C_{1,2}+1\right), \\
& C_{1,2}^{(2)}=C_{1,2} \cup-\left(A_{1,2}+1\right), \\
& B_{1,2}^{(2)}=B_{1,2}^{+} \cup-\left(B_{1,2}^{+}+1\right) .
\end{aligned}
$$

Corollary 43. The sets $2 S_{1}$ and $S_{1}^{(2)}:=2 S_{1} \cup-\left(2 S_{1}+1\right)$ are midpoint-free subsets of $\mathbb{Z}$ with endpoint sets satisfying $A_{1,2} \cup$ $C_{1,2}=2 \mathbb{Z} \backslash 2 S_{1}$ and $A_{1,2}^{(2)} \cup C_{1,2}^{(2)}=\mathbb{Z} \backslash S_{1}^{(2)}$. 
The balance point sets of $S_{1}^{(3)}:=3 S_{1} \cup\left(3 S_{1}+1\right)$ can now be considered. Let

$$
\begin{gathered}
A_{1,3}^{+}:=A\left(S_{1}^{(3)}, \mathbb{Z}^{+}\right), \quad B_{1,3}^{+}:=B\left(S_{1}^{(3)}, \mathbb{Z}^{+}\right), \\
C_{1,3}^{+}:=C\left(S_{1}^{(3)}, \mathbb{Z}^{+}\right), \\
A_{1,3}:=A\left(S_{1}^{(3)}, \mathbb{Z}\right), \quad B_{1,3}:=B\left(S_{1}^{(3)}, \mathbb{Z}\right), \\
C_{1,3}:=C\left(S_{1}^{(3)}, \mathbb{Z}\right) .
\end{gathered}
$$

If $a+x=2 b$ and $a, b \in S_{1}:=\mathbb{Z}_{4}^{+}(0,1)$, the conditions

$$
3 a+r, 3 b+s \in S_{1}^{(3)}=3 S_{1} \cup\left(3 S_{1}+1\right)
$$

and $(3 a+r)+(3 x+t)=2(3 b+s)$ are satisfied by the triples $(r, s, t)=(0,0,0),(1,1,1),(1,0,-1),(0,1,2)$. Hence, if $x \in A_{1}^{+} \cup C_{1}^{+}$then $3 x+\{-1,0,1,2\} \subset A_{1,3}^{+} \cup C_{1,3}^{+}$. Such observations yield the following results.

Theorem 44. The subset $S_{1}^{(3)}:=3 S_{1} \cup\left(3 S_{1}+1\right) \subset \mathbb{Z}^{+}$is midpoint-free and has balance point sets satisfying

$$
\begin{gathered}
A_{1,3}^{+} \cup C_{1,3}^{+}=\mathbb{Z}^{+} \backslash S_{1}^{(3)}, \\
B_{1,3}^{+}=3 B_{1}^{+} \cup\left(3 B_{1}^{+}+1\right) \cup\left(3 B_{1}^{\sim}-1\right),
\end{gathered}
$$

where $B_{1}^{\sim}:=\left(B_{1}^{+} \cup\{1\}\right) \backslash 2 \mathbb{Z}^{+}$.

Corollary 45. The subset $S_{1}^{(3)}:=3 S_{1} \cup\left(3 S_{1}+1\right) \subset \mathbb{Z}$ is midpoint-free and has balance point sets satisfying

$$
A_{1,3}=A_{1,3}^{+} \cup \mathbb{Z}^{-}, \quad B_{1,3}=B_{1,3}^{+}, \quad C_{1,3}=C_{1,3}^{+} .
$$

\section{The Midpoint-Free Set $\mathbb{Z}_{7}^{+}(0,1,3)$}

Next consider the set

$$
S_{2}:=\mathbb{Z}_{7}^{+}(0,1,3)=\{0,1,3,7,8,10,21,22,24,49, \ldots\} .
$$

At the time of writing, no corresponding sequence appears in OEIS [5]. However, Corollary 10 asserts that it is midpointfree, and it will be shown that $S_{2}$ is in fact a maximal midpoint-free subset of $\mathbb{Z}$. It will follow from Theorems 4 and 6 that

$$
S_{2}^{(2)}:=2 S_{1} \cup-\left(2 S_{1}+1\right), \quad S_{2}^{(3)}:=3 S_{2} \cup\left(3 S_{2}+1\right)
$$

are maximal midpoint-free subsets of $\mathbb{Z}$.

The methods used in earlier sections are again applicable, so fewer details are now required. Following earlier practice, let

$$
\begin{gathered}
A_{2}^{+}:=A\left(S_{2}, \mathbb{Z}^{+}\right), \quad B_{2}^{+}:=B\left(S_{2}, \mathbb{Z}^{+}\right), \\
C_{2}^{+}:=C\left(S_{2}, \mathbb{Z}^{+}\right), \\
A_{2}:=A\left(S_{2}, \mathbb{Z}\right), \quad B_{2}:=B\left(S_{2}, \mathbb{Z}\right), \\
C_{2}:=C\left(S_{2}, \mathbb{Z}\right) .
\end{gathered}
$$

Theorem 46. The midpoint-free subset $S_{2}:=\mathbb{Z}_{7}^{+}(0,1,3) \subset \mathbb{Z}^{+}$ has endpoint sets satisfying $A_{2}^{+} \cup C_{2}^{+}=\mathbb{Z}^{+} \backslash S_{2}$.

Proof. Given $x \in \mathbb{Z}^{+} \backslash S_{2}$, we seek $y, b \in S_{2}$ such that $x+y=2 b$. Any solution has $x \neq y$. Base 7 computation requires

$$
\llbracket x \rrbracket_{7, i}+\llbracket y \rrbracket_{7, i}+\delta_{i}=2 \llbracket b \rrbracket_{7, i}+7 \delta_{i+1}
$$

for $i \geq 0$, with appropriate carry-overs $\delta_{i} \in\{0,1\}$ beginning with $\delta_{0}=0$. All base 7 digits of $y, b$ are determined by

$$
\begin{gathered}
\llbracket x \rrbracket_{7, i}+\delta_{i} \in\{0,7\} \Longrightarrow \llbracket y \rrbracket_{7, i}=\llbracket b \rrbracket_{7, i}=0, \\
\llbracket x \rrbracket_{7, i}+\delta_{i}=1 \Longrightarrow \llbracket y \rrbracket_{7, i}=\llbracket b \rrbracket_{7, i}=1, \\
\llbracket x \rrbracket_{7, i}+\delta_{i}=2 \Longrightarrow \llbracket y \rrbracket_{7, i}=0, \quad \llbracket b \rrbracket_{7, i}=1, \\
\llbracket x \rrbracket_{7, i}+\delta_{i}=3 \Longrightarrow \llbracket y \rrbracket_{7, i}=\llbracket b \rrbracket_{7, i}=3, \\
\llbracket x \rrbracket_{7, i}+\delta_{i}=4 \Longrightarrow \llbracket y \rrbracket_{7, i}=3, \quad \llbracket b \rrbracket_{7, i}=0, \\
\llbracket x \rrbracket_{7, i}+\delta_{i}=5 \Longrightarrow \llbracket y \rrbracket_{7, i}=1, \quad \llbracket b \rrbracket_{7, i}=3, \\
\llbracket x \rrbracket_{7, i}+\delta_{i}=6 \Longrightarrow \llbracket y \rrbracket_{7, i}=0, \quad \llbracket b \rrbracket_{7, i}=3 .
\end{gathered}
$$

It is straightforward to verify that all requirements are satisfied.

Corollary 47 . The set $\mathbb{Z}_{7}^{+}(0,1,3)$ is a maximal midpoint-free subset of $\mathbb{Z}^{+}$.

Corollary 48. For $S_{2}:=\mathbb{Z}_{7}^{+}(0,1,3)$, any $x \in \mathbb{Z}^{+} \backslash S_{2}$ has endpoint multiplicity

$$
\alpha\left(x, S_{2}\right)+\gamma\left(x, S_{2}\right)=1 .
$$

Corollary 49. The midpoint set $B_{2}^{+}$for $S_{2}:=\mathbb{Z}_{7}^{+}(0,1,3)$ in $\mathbb{Z}^{+}$ comprises those integers $x \in \mathbb{Z}^{+} \backslash S_{2}$ with $2 x \in \mathbb{Z}_{7}^{+}([0,6] \backslash\{5\})$. Any such $x$ has midpoint multiplicity $\beta\left(x, S_{2}\right)=2^{k-1}$, where $k>0$ is the number of digits in $(2 x)_{7}$ belonging to $\{1,3,4\}$.

Proof. Given $x \in \mathbb{Z}^{+} \backslash S_{2}$, we seek $a, c \in S_{2}$ such that $a+$ $c=2 x$. Necessarily, any solution has $a \neq x$, so $a \neq c$ holds. Base 7 arithmetic requires

$$
\llbracket a \rrbracket_{7, i}+\llbracket c \rrbracket_{7, i}=\llbracket 2 x \rrbracket_{7, i}
$$

for every $i \geq 0$. This forces $\llbracket 2 x \rrbracket_{7, i} \in[0,6] \backslash\{5\}$. Since $x \notin$ $S_{2}$ there is an integer $j \geq 0$ such that $\llbracket 2 x \rrbracket_{7, j} \notin\{0,2,6\}$ but $\llbracket 2 x \rrbracket_{7, i} \in\{0,2,6\}$ for all $i>j$. Specify $a, c$ by

$$
\begin{aligned}
\llbracket 2 x \rrbracket_{7, i} \in\{0,2,6\} & \Longrightarrow \llbracket a \rrbracket_{7, i}=\llbracket c \rrbracket_{7, i}=\frac{1}{2} \llbracket 2 x \rrbracket_{7, i} \\
\llbracket 2 x \rrbracket_{7, i}=1 & \Longrightarrow\left\{\llbracket a \rrbracket_{7, i}, \llbracket c \rrbracket_{7, i}\right\}=\{0,1\}, \\
\llbracket 2 x \rrbracket_{7, i}=3 & \Longrightarrow\left\{\llbracket a \rrbracket_{7, i}, \llbracket c \rrbracket_{7, i}\right\}=\{0,3\}, \\
\llbracket 2 x \rrbracket_{7, i}=4 & \Longrightarrow\left\{\llbracket a \rrbracket_{7, i}, \llbracket c \rrbracket_{7, i}\right\}=\{1,3\} .
\end{aligned}
$$

With $\llbracket a \rrbracket_{4, j}<\llbracket c \rrbracket_{4, j}$, evidently all solutions satisfy $a+c=2 x$ and $a<c$. 
The base 7 representation of the integer $u_{7, n}:=\left(7^{n}-1\right) / 6$ is a block of $n$ digits, all equal to 1 . Thus $u_{7, n}$ is a base 7 repunit [6]. (See A023000 in OEIS [5].) For any $x \in \mathbb{Z}^{+}$let $\left.x\right|_{7, k}$ denote the integer $y$ resulting from $(x)_{7}$ by deleting all but the last $k$ base 7 digits:

$$
\begin{array}{cl}
\llbracket y \rrbracket_{7, i}=0 & \text { for } i \geq k, \\
\llbracket y \rrbracket_{7, i}=\llbracket x \rrbracket_{7, i} & \text { for } k>i \geq 0 .
\end{array}
$$

An alternative characterization of the midpoint set $B_{2}^{+}$is that it comprises every $x \in \mathbb{Z}^{+}$with $\llbracket x \rrbracket_{7, i} \in\{2,4,5,6\}$ for at least one $i \geq 0$ and

$$
\begin{aligned}
& \llbracket x \rrbracket_{7, k}=\left.2 \Longrightarrow x\right|_{7, k} \leq 3 u_{7, k}, \\
& \llbracket x \rrbracket_{7, k}=\left.6 \Longrightarrow x\right|_{7, k}>3 u_{7, k} .
\end{aligned}
$$

Corollary 50. The midpoint-free subset $S_{2}:=\mathbb{Z}_{7}^{+}(0,1,3) \subset \mathbb{Z}$ has endpoint sets satisfying $A_{2}=A_{2}^{+} \cup \mathbb{Z}^{-}, B_{2}=B_{2}^{+}, C_{2}=C_{2}^{+}$.

Proof. Given $-x \in \mathbb{Z}^{-}$, we seek $b, c \in S_{2}$ such that $x+2 b=c$. Clearly $-x \neq c$. Base 7 computation requires

$$
\llbracket x \rrbracket_{7, i}+2 \llbracket b \rrbracket_{7, i}+\delta_{i}=\llbracket c \rrbracket_{7, i}+7 \delta_{i+1}
$$

for $i \geq 0$, with appropriate carry-overs $\delta_{i} \in\{0,1\}$ beginning with $\delta_{0}=0$. All base 7 digits of $b, c$ are determined by

$$
\begin{gathered}
\llbracket x \rrbracket_{7, i}+\delta_{i} \in\{0,7\} \Longrightarrow \llbracket b \rrbracket_{7, i}=\llbracket c \rrbracket_{7, i}=0, \\
\llbracket x \rrbracket_{7, i}+\delta_{i}=1 \Longrightarrow\left(\llbracket b \rrbracket_{7, i}, \llbracket c \rrbracket_{7, i}\right) \in\{(0,1),(1,3),(3,0)\}, \\
\llbracket x \rrbracket_{7, i}+\delta_{i}=2 \Longrightarrow \llbracket b \rrbracket_{7, i}=3, \quad \llbracket c \rrbracket_{7, i}=1, \\
\llbracket x \rrbracket_{7, i}+\delta_{i}=3 \Longrightarrow \llbracket b \rrbracket_{7, i}=0, \quad \llbracket c \rrbracket_{7, i}=3, \\
\llbracket x \rrbracket_{7, i}+\delta_{i}=4 \Longrightarrow \llbracket b \rrbracket_{7, i}=\llbracket c \rrbracket_{7, i}=3, \\
\llbracket x \rrbracket_{7, i}+\delta_{i}=5 \Longrightarrow \llbracket b \rrbracket_{7, i}=1, \quad \llbracket c \rrbracket_{7, i}=0, \\
\llbracket x \rrbracket_{7, i}+\delta_{i}=6 \Longrightarrow \llbracket b \rrbracket_{7, i}=\llbracket c \rrbracket_{7, i}=1 .
\end{gathered}
$$

Let $x$ have leading digit in position $h \geq 0$. If $\llbracket b \rrbracket_{7, h}<$ $\llbracket c \rrbracket_{7, h}$ then $\delta_{h+1}=0$ so $b<c$, since $b, c$ have leading digits in position $h$. All requirements are satisfied.

Now suppose that $\llbracket b \rrbracket_{7, h} \geq \llbracket c \rrbracket_{7, h}$. Then in every case $\delta_{h+1}=1$, and

$$
\left(\llbracket b \rrbracket_{7, h+1}, \llbracket c \rrbracket_{7, h+1}\right) \in\{(0,1),(1,3),(3,0)\} .
$$

The first two options here ensure that $\delta_{h+2}=0$ and $b, c$ have leading digits in position $h+1$, and $b<c$. However, $\llbracket b \rrbracket_{7, h+1}>$ $\llbracket c \rrbracket_{7, h+1}$ holds if the third option is chosen, and then $\delta_{h+2}=1$, so

$$
\left(\llbracket b \rrbracket_{7, h+2}, \llbracket c \rrbracket_{7, h+2}\right) \in\{(0,1),(1,3),(3,0)\} .
$$

This behaviour can be iterated any finite number of times but must terminate at some stage in order to determine integers $b, c$. We may choose any integer $k>0$ and assign

$$
\left(\llbracket b \rrbracket_{7, h+k}, \llbracket c \rrbracket_{7, h+k}\right) \in\{(0,1),(1,3)\},
$$

with $\llbracket b \rrbracket_{7, h+i}=3, \llbracket c \rrbracket_{7, h+i}=0$ if $0<i<k$. The leading digits of $b, c$ are in position $h+k$, so $b<c$. Once again, all requirements are satisfied.

Corollary 51. The set $\mathbb{Z}_{7}^{+}(0,1,3)$ is a maximal midpoint-free subset of $\mathbb{Z}$.

The proof of Corollary 50 implies a surprising result.

Corollary 52. Let $\llbracket x \rrbracket_{7, h} \in\{4,5,6\}$ be the leading base 7 digit of $x \in \mathbb{Z}^{+}$. Then $-x$ is a lower endpoint for the midpoint-free subset $S_{2}:=\mathbb{Z}_{7}^{+}(0,1,3) \subset \mathbb{Z}$ with multiplicity $\alpha\left(-x, S_{2}\right)=\aleph_{0}$.

Proof. The condition $\llbracket x \rrbracket_{7, h} \in\{4,5,6\}$ necessitates $\llbracket b \rrbracket_{7, h} \geq$ $\llbracket c \rrbracket_{7, h}$. Let $b, c \in S_{2}$ be a solution to $x+2 b=c$ with leading digits $\llbracket b \rrbracket_{7, h+1}=1, \llbracket c \rrbracket_{7, h+1}=3$. For $g>h$, replace the leading 1 of $(b)_{7}$ by a block comprising a leading digit 1 followed by $g-h$ digits all equal to 3 . This yields a new solution with the leading 3 of $(c)_{7}$ replaced by a block comprising a leading digit 3 followed by $g-h$ digits all equal to 0 . Using base 7 rep-units, this yields

$$
\begin{gathered}
x+2\left(b-7^{h+1}+3\left(u_{7, g+1}-u_{7, h+1}\right)+7^{g+1}\right) \\
=c-3 \cdot 7^{h+1}+3 \cdot 7^{g+1},
\end{gathered}
$$

since $6\left(u_{7, g+1}-u_{7, h+1}\right)=7^{g+1}-7^{h+1}$. Thus,

$$
\left(-x, b+9\left(u_{7, g+1}-u_{7, h+1}\right), c+18\left(u_{7, g+1}-u_{7, h+1}\right)\right) \in \Lambda(\mathbb{Z})
$$

for every $g>h$, so there are infinitely many triples in $\Lambda(\mathbb{Z})$ having $-x$ as lower endpoint, with midpoint and upper endpoint in $S_{2}$.

\section{Closing Remarks}

Remark 1. We have seen that the midpoint-free set $S_{5}:=$ $\mathbb{Z}_{5}^{+}(0,1)$ is not maximal in $\mathbb{Z}^{+}$. The smallest member of $E\left(S_{5}, \mathbb{Z}^{+}\right)$is 8 , confirming Corollary 15 when $m=5$. If $S_{5} \subset$ $T \subset \mathbb{Z}^{+}$and $T$ is a maximal midpoint-free subset of $\mathbb{Z}^{+}$then

$$
T \backslash S_{5} \subset E\left(S_{5}, \mathbb{Z}^{+}\right)
$$

This raises some intriguing open questions. For which subsets $X \subset E\left(S_{5}, \mathbb{Z}^{+}\right)$is $S_{5} \cup X$ a maximal midpoint-free subset of $\mathbb{Z}^{+}$? What is the greedy subset $S_{5}^{*} \subset E\left(S_{5}, \mathbb{Z}^{+}\right)$which makes $S_{5} \cup S_{5}^{*}$ a maximal midpoint-free subset of $\mathbb{Z}^{+}$?

Note that $S_{5} \cup\{x\}$ is midpoint-free for any $x \in E\left(S_{5}, \mathbb{Z}^{+}\right)$, but $S_{5} \cup\{x, y\}$ is not always midpoint-free if $x, y \in E\left(S_{5}, \mathbb{Z}^{+}\right)$. For instance, the midpoint triple $(8,25,42)$ comprises $25 \in S_{5}$ and $8,42 \in E\left(S_{5}, \mathbb{Z}^{+}\right)$.

Remark 2. Consider the balance points of $S_{5}:=\mathbb{Z}_{5}^{+}(0,1)$. Using notation defined after the proof of Corollary 49 , along with base 5 rep-units $u_{5, k}$ (see A003463 in OEIS [5]), for 
$x \in \mathbb{Z}^{+} \backslash S_{5}$ we have $x \in A\left(S_{5}, \mathbb{Z}^{+}\right) \cup C\left(S_{5}, \mathbb{Z}^{+}\right)$precisely when

$$
\begin{aligned}
& \llbracket x \rrbracket_{5, k}=\left.2 \Longrightarrow x\right|_{5, k} \leq 3 u_{5, k}, \\
& \llbracket x \rrbracket_{5, k}=\left.3 \Longrightarrow x\right|_{5, k}>3 u_{5, k} .
\end{aligned}
$$

Hence, in particular, $\mathbb{Z}_{5}^{+}(0,1,4) \backslash S_{5} \subset A\left(S_{5}, \mathbb{Z}^{+}\right) \cup C\left(S_{5}, \mathbb{Z}^{+}\right)$. Similarly, if $x \in \mathbb{Z}^{+} \backslash S_{5}$ then $x \in B\left(S_{5}, \mathbb{Z}^{+}\right)$precisely when $\llbracket x \rrbracket_{5, i} \neq 4$ for all $i \geq 0$, and

$$
\begin{aligned}
& \llbracket x \rrbracket_{5, k}=\left.1 \Longrightarrow x\right|_{5, k} \leq 2 u_{5, k}, \\
& \llbracket x \rrbracket_{5, k}=\left.2 \Longrightarrow x\right|_{5, k}>2 u_{5, k} .
\end{aligned}
$$

In particular, $\mathbb{Z}_{5}^{+}(0,3) \backslash\{0\} \subset B\left(S_{5}, \mathbb{Z}^{+}\right)$.

If $x \in \mathbb{Z}^{+} \backslash S_{5}$ is eccentric for $S_{5}$, the digit configuration $(x)_{5}$ violates each of these conditions. If $x<5^{h}$ then $\left(x+5^{h} y\right)_{5}$ contains the same digit configuration for every $y \in \mathbb{Z}^{+}$, so $x+5^{h} y \in E\left(S_{5}, \mathbb{Z}^{+}\right)$. Every eccentric point $x$ is the lower endpoint of infinitely many midpoint triples

$$
\left(x, x+5^{h} y, x+2 \cdot 5^{h} y\right) \in \Lambda\left(E\left(S_{5}, \mathbb{Z}^{+}\right)\right),
$$

so $E\left(S_{5}, \mathbb{Z}^{+}\right)$is densely packed with midpoint triples.

Remark 3. The set $T_{5}:=\mathbb{Z}_{5}^{+}(0,1,3)$ is not midpoint-free, since $(0,3,6) \in \Lambda\left(T_{5}\right)$. The digit 3 is responsible for "most" members of $T_{5}$ being midpoints, since it can be shown that $B\left(T_{5}, \mathbb{Z}^{+}\right)=\mathbb{Z}^{+} \backslash S_{5}$. Moreover, for any $x \in \mathbb{Z}^{+} \backslash S_{5}$ there is at least one triple $(a, x, c) \in \Lambda\left(\mathbb{Z}^{+}\right)$with $a \in S_{5}$ and $c \in T_{5}$.

It can be shown that $A\left(T_{5}, \mathbb{Z}^{+}\right) \cup C\left(T_{5}, \mathbb{Z}^{+}\right)=\mathbb{Z}^{+}$and $\mathbb{Z}^{-} \subset A\left(T_{5}, \mathbb{Z}\right)$, so no integer is eccentric for $T_{5}$. Hence there are maximal midpoint-free subsets $T \subset \mathbb{Z}$ such that $S_{5} \subset T \subset$ $T_{5}$. What is the greedy subset $T_{5}^{*} \subset T_{5} \backslash S_{5}$ which makes $S_{5} \cup T_{5}^{*}$ a maximal midpoint-free subset of $\mathbb{Z}$ ?

Note also that if $(x, b, c) \in \Lambda(\mathbb{Z})$ and $b, c \in T_{5}$, then

$$
\left(x, b+3 \cdot 5^{h}, c+6 \cdot 5^{h}\right) \in \Lambda(\mathbb{Z})
$$

and $b+3 \cdot 5^{h}, c+6 \cdot 5^{h} \in T_{5}$ for every sufficiently large $h \in \mathbb{Z}^{+}$. It follows that every $x \in \mathbb{Z}$ has lower endpoint multiplicity $\alpha\left(x, T_{5}\right)=\aleph_{0}$.

Remark 4. The set $T_{6}:=\mathbb{Z}_{6}^{+}(0,1,3)$ is not midpoint-free. Once again, the digit 3 is responsible for "most" members of $T_{6}$ being midpoints, since it can be shown that $T_{6} \backslash S_{6} \subset$ $B\left(T_{6}, \mathbb{Z}^{+}\right)$, where $S_{6}:=\mathbb{Z}_{6}^{+}(0,1)$. However, $E\left(T_{6}, \mathbb{Z}^{+}\right) \neq \emptyset$. For example, it is easily verified that $\left\{x \in \mathbb{Z}^{+} \mid \llbracket x \rrbracket_{6,0}=4\right.$, $\left.\llbracket x \rrbracket_{6,1}=2\right\} \subset E\left(T_{6}, \mathbb{Z}^{+}\right)$. Thus there is no maximal midpointfree subset $T \subset \mathbb{Z}$ that satisfies $S_{6} \subset T \subseteq T_{6}$. What is the greedy subset $S_{6}^{*} \subset E\left(S_{6}, \mathbb{Z}^{+}\right)$which makes $S_{6} \cup S_{6}^{*}$ a maximal midpoint-free subset of $\mathbb{Z}^{+}$?

Remark 5. The midpoint-free set $S_{8}:=\mathbb{Z}_{8}^{+}(0,1,3)$ is not maximal in $\mathbb{Z}^{+}$. Indeed, confirming Corollary 16 when $m=8$, the smallest member of $E\left(S_{8}, \mathbb{Z}^{+}\right)$is 20 . In fact,

$$
\left\{x \in \mathbb{Z}^{+} \llbracket x \rrbracket_{8,0}=4, \llbracket x \rrbracket_{8,1}=2\right\} \subset E\left(S_{8}, \mathbb{Z}^{+}\right) .
$$

For $x \in \mathbb{Z}^{+} \backslash S_{8}$ it can be shown that $x \in A\left(S_{8}, \mathbb{Z}^{+}\right) \cup$ $C\left(S_{8}, \mathbb{Z}^{+}\right)$precisely when

$$
\begin{aligned}
& \llbracket x \rrbracket_{8, k}=\left.3 \Longrightarrow x\right|_{8, k} \leq 4 u_{8, k}, \\
& \llbracket x \rrbracket_{8, k}=\left.4 \Longrightarrow x\right|_{8, k}>4 u_{8, k}
\end{aligned}
$$

so, in particular, $\mathbb{Z}_{8}^{+}([0,3]) \backslash S_{8} \subset A\left(S_{8}, \mathbb{Z}^{+}\right) \cup C\left(S_{8}, \mathbb{Z}^{+}\right)$. (For base 8 rep-units $u_{8, k}$, see A023001 in OEIS [5].) Again, if $x \in$ $\mathbb{Z}^{+} \backslash S_{8}$ then $x \in B\left(S_{8}, \mathbb{Z}^{+}\right)$precisely when

$$
\left.\llbracket x \rrbracket_{8, k} \in\{2,3,6,7\} \Longrightarrow x\right|_{8, k} \leq 3 u_{8, k}
$$

so, in particular, $\mathbb{Z}_{8}^{+}([0,3]) \backslash S_{8} \subset B\left(S_{8}, \mathbb{Z}^{+}\right)$. What is the greedy subset $S_{8}^{*} \subset E\left(S_{8}, \mathbb{Z}^{+}\right)$which makes $S_{8} \cup S_{8}^{*}$ a maximal midpoint-free subset of $\mathbb{Z}^{+}$?

Remark 6. The set $S_{9}:=\mathbb{Z}_{9}^{+}(0,1,3,4)$ is a maximal midpointfree subset of $\mathbb{Z}^{+}$since $\mathbb{Z}_{9}^{+}(0,1,3,4)=\mathbb{Z}_{3}^{+}(0,1)$. This follows immediately from the observation that

$$
(3 r+s) 9^{n}=r 3^{2 n+1}+s 3^{2 n} \quad \text { when } r, s \in\{0,1\}, n \geq 0 .
$$

More generally, $\mathbb{Z}_{m}^{+}(D)=\mathbb{Z}_{3}^{+}(0,1)$ when $m=3^{n}$ and $D=$ $S_{0} \cap\left[0, u_{3, n}\right]$ for any positive integer $n$.

Remark 7. The midpoint-free sets $S_{m}:=\mathbb{Z}_{m}^{+}(0,1,3,4)$ are maximal in $\mathbb{Z}^{+}$for each $m \in[9,13]$, and the endpoint sets satisfy $A\left(S_{m}, \mathbb{Z}^{+}\right) \cup C\left(S_{m}, \mathbb{Z}^{+}\right)=\mathbb{Z}^{+} \backslash S_{m}$. Thus $S_{m}^{(2)}:=$ $2 S_{m} \cup-\left(2 S_{m}+1\right)$ and $S_{m}^{(3)}:=3 S_{m} \cup\left(3 S_{m}+1\right)$ are maximal midpoint-free subsets of $\mathbb{Z}$ when $m \in[9,13]$, by Theorems 4 and 6. However, the midpoint-free set $S_{14}$ is not maximal in $\mathbb{Z}^{+}$since, confirming Corollary 17 when $m=14$, the smallest member of $E\left(S_{14}, \mathbb{Z}^{+}\right)$is 65 . What is the greedy subset $S_{14}^{*} \subset$ $E\left(S_{14}, \mathbb{Z}^{+}\right)$which makes $S_{14} \cup S_{14}^{*}$ a maximal midpoint-free subset of $\mathbb{Z}^{+}$?

\section{Conflict of Interests}

The author declares that there is no conflict of interests regarding the publication of this paper.

\section{Acknowledgment}

Thanks are due to James MacDougall for suggesting the problem of determining multiplicity of solutions for the greedy midpoint-free sequence $S_{0}$.

\section{References}

[1] R. K. Guy, Unsolved Problems in Number Theory, Springer, New York, NY, USA, 3rd edition, 2004.

[2] J. Dybizbański, "Sequences containing no 3-term arithmetic progressions," Electronic Journal of Combinatorics, vol. 19, no. 2, pp. 1-5, 2012.

[3] R. B. Eggleton, "Midpoint-free subsets of the real numbers," International Journal of Combinatorics, vol. 2014, Article ID 214637, 8 pages, 2014. 
[4] P. Erdős and M. Lewin, “On some sequences of integers," Journal of the London Mathematical Society, vol. 28, pp. 104-109, 1936.

[5] N. J. A. Sloane, OEIS-Online Encyclopedia of Integer Sequences, https://oeis.org/.

[6] S. Yates, "Prime divisors of repunits," Journal of Recreational Mathematics, vol. 8, no. 1, pp. 33-38, 1975. 


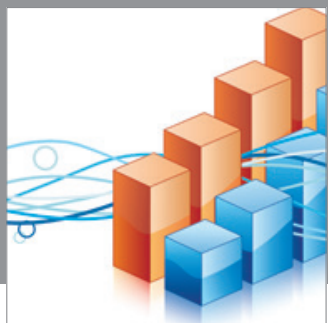

Advances in

Operations Research

mansans

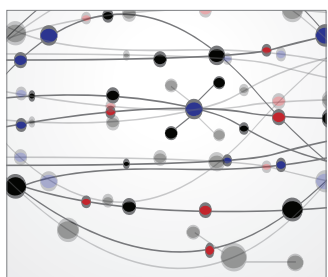

The Scientific World Journal
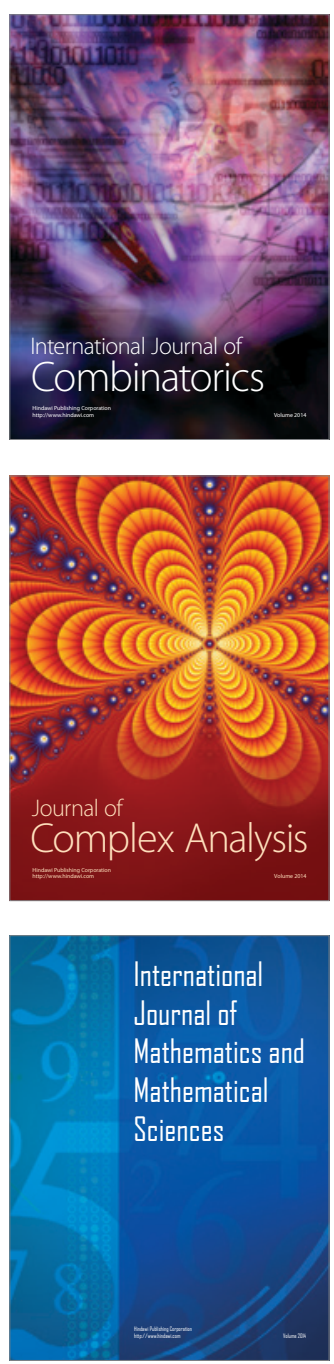
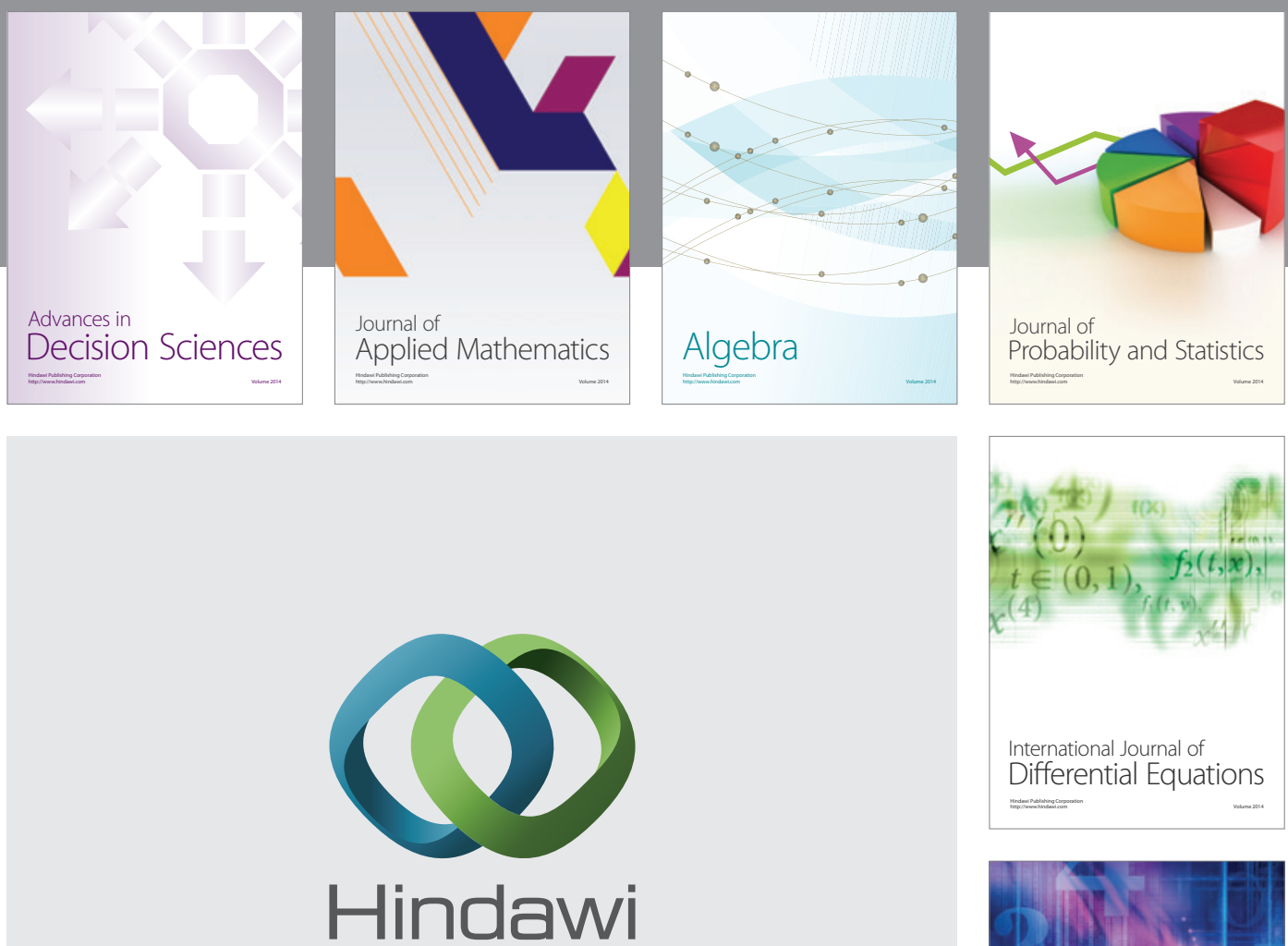

Submit your manuscripts at http://www.hindawi.com
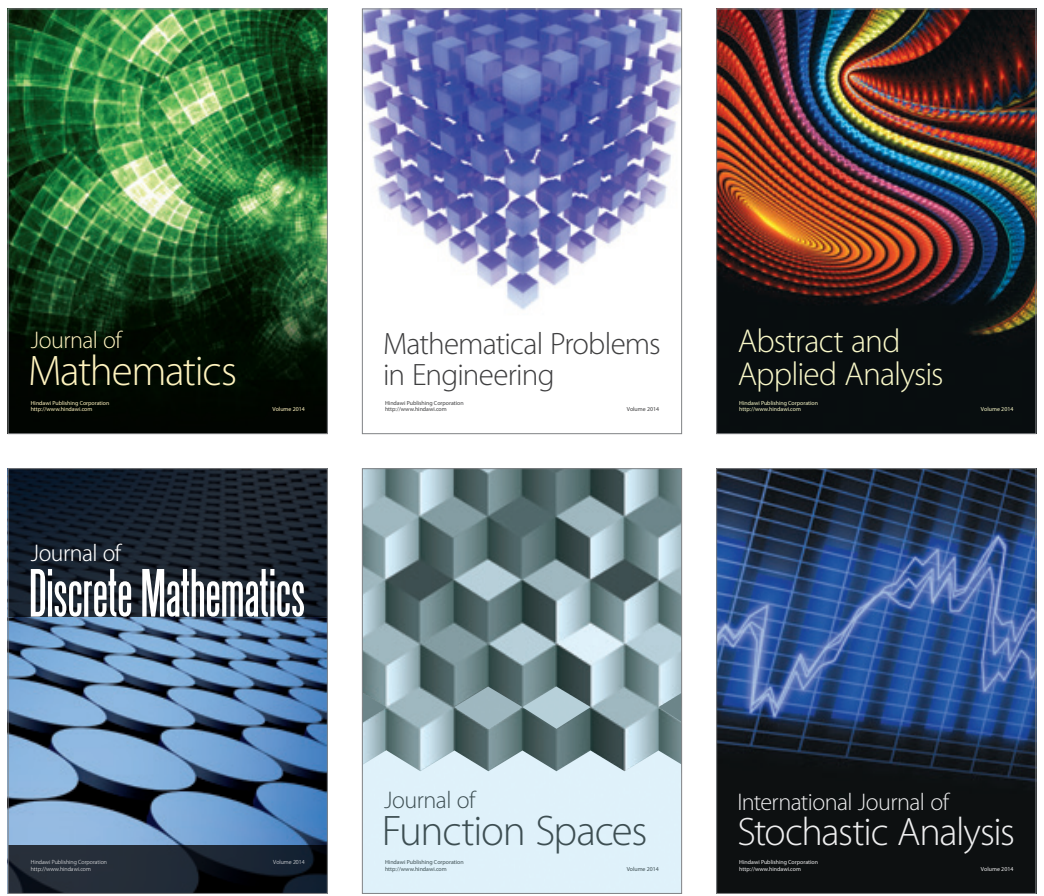

Journal of

Function Spaces

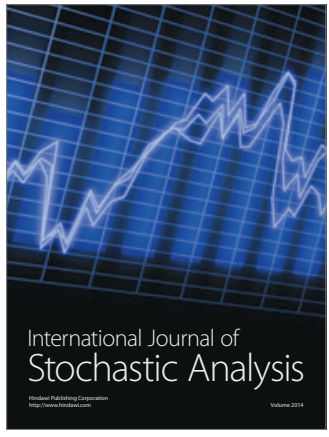

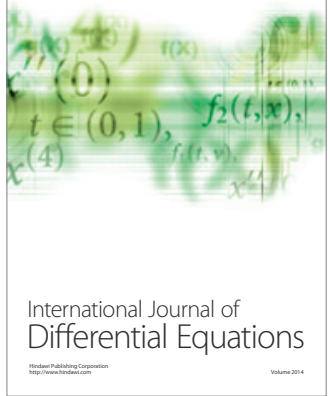
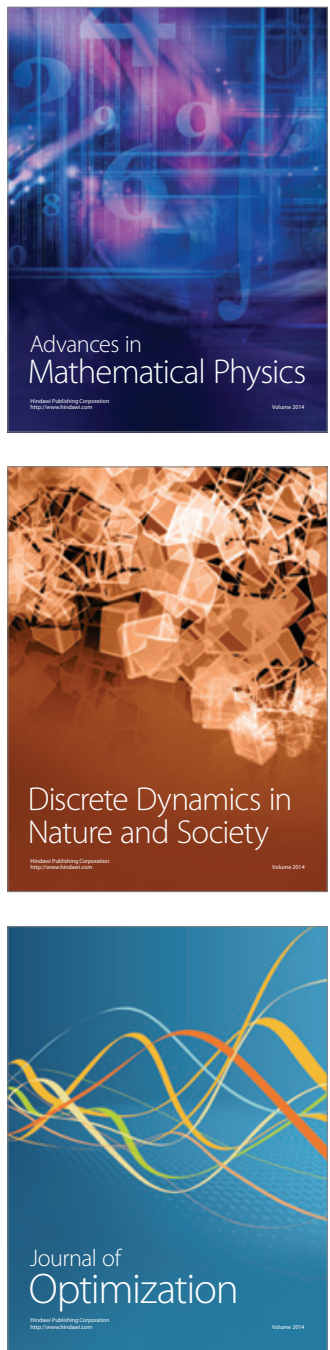